\title{
LA RICOLONIZZAZIONE DELL'ANTARTIDE: STORIE DI PINGUINI E FOCHE, SENTINELLE DEI CAMBIAMENTI CLIMATICI NEGLI ULTIMI 10 MILA ANNI
}

\author{
CARLO BARONI (*) \\ Nota presentata dal m.e. Giuseppe Orombelli \\ (Adunanza del 1 febbraio 2018)
}

SunTO. - Nel Pleistocene superiore l'espansione del sistema glaciale antartico ha invaso la fascia costiera costringendo pinguini, foche e altri animali che nidificavano nelle aree deglaciate a migrare verso aree rifugio poste a latitudini più settentrionali. La deglaciazione che è seguita all'ultimo periodo di massima espansione glaciale, nel settore del Mare di Ross, si è completata intorno a 8000 anni fa, liberando le coste antartiche che sono state ri-colonizzate da pinguini di Adelia ed elefanti marini. Queste due specie hanno convissuto, anche alternandosi, nelle aree deglaciate della fascia costiera per diversi millenni. Nei suoli ornitogenici delle colonie di pinguini abbandonate e nei siti di riproduzione degli elefanti marini recentemente scoperti sulle spiagge emerse è conservato un importante patrimonio di resti organici che ci permette di studiare diversi aspetti della storia ambientale recente. Le fasi della colonizzazione e della coesistenza tra pinguini ed elefanti marini sono molto articolate e riflettono le variazioni climaticoambientali che hanno caratterizzato lo scenario antartico per diverse migliaia di anni. Pinguini ed elefanti marini, sensibili sentinelle dell'ecosistema antartico stanno anche adesso recitando un ruolo di rilievo nello scenario dell'Oceano Meridionale, interpretando la parte di veri e propri campanelli d'allarme dei cambiamenti climatici.

$* * *$

ABSTRACT. - The re-colonization of Antarctica: stories of penguins and seals, sentinels of climate change in the last ten thousand years. During the Late Pleistocene the

(*) Istituto Lombardo Accademia di Scienze e Lettere, Milano. Dipartimento di Scienze della Terra, Università di Pisa. CNR-IGG Istituto di Geoscienze e Georisorse, Pisa, Italy. E-mail: carlo.baroni@unipi.it 
Antarctic glacial system expanded and advanced on the coastal margin of the continent forcing penguins, seals and other animals nesting in the deglaciated areas to migrate toward refuge areas located at northern latitudes. The deglaciation that followed the last glacial maximum, in the Ross Sea area, was accomplished around 8000 years ago, freeing the Antarctic coasts that were re-colonized by Adélie penguins and elephant seals. These two species have coexisted, even alternating, in the deglaciated areas of the coastal strip for several millennia. In the ornithogenic soils of the abandoned penguin colonies and in the breeding sites of the elephant seals, recently discovered on the Holocene raised beaches, a precious heritage of organic remains is preserved, allowing us to study different aspects of recent environmental history. The phases of colonization and coexistence between penguins and elephant seals are very complex and reflect the climatic-environmental variations that have characterized the Antarctic scenario for several thousand of years. Penguins and elephant seals, sensitive sentinels of the Antarctic ecosystem, are now playing an important role in the Southern Ocean scenario, interpreting the dramatic role of bellwether of climate change.

\section{INTRODUZIONE}

Nelle regioni polari, dominate dalla criosfera, i processi glaciali e periglaciali costituiscono i principali e più efficaci agenti morfogenetici, in grado di scolpire spettacolari forme del rilievo che rendono unici i paesaggi di queste regioni. I sistemi glaciali che si sviluppano attualmente sull'Antartide (circa 13.5*10 $\mathrm{km}^{2}$ ) e sulla Groenlandia (circa 1.7 $* 10^{6} \mathrm{~km}^{2}$ ) comprendono il $96 \%$ della superficie glacializzata del nostro Pianeta, trattenendo circa il $99 \%$ del volume di ghiaccio totale (WGMS). L'enorme quantità di ghiaccio immagazzinata nelle calotte antartiche (circa $26.6 * 10^{6} \mathrm{~km}^{3}$ ) e in Groenlandia (circa $2.85 * 10^{6} \mathrm{~km}^{3}$ ) è in grado di condizionare il livello medio del mare a scala globale: l'eventuale fusione delle calotte rilascerebbe una quantità d'acqua in grado di determinare un innalzamento del livello del mare di oltre $68 \mathrm{~m}$ $(61 \mathrm{~m}$ circa dalla fusione delle calotte antartiche, $7.2 \mathrm{~m}$ dalla fusione della calotta groenlandese; Morlighem et al., 2020).

Oltre a condizionare le variazioni del livello del mare, i sistemi glaciali che si sviluppano nelle regioni polari, guidano la circolazione oceanica e il relativo trasferimento di calore attraverso l'attivazione o la disattivazione della circolazione termoalina (conveyor belt) e controllano i livelli di gas serra attraverso i processi di emissione/assorbimento di $\mathrm{CO}_{2}$ e $\mathrm{CH}_{4}$. Le regioni polari svolgono un ruolo di primo piano nel bilancio energetico planetario da almeno $34 \mathrm{Ma}$ (Eocene-Oligocene), 
cioè dall'epoca in cui si sviluppò la prima calotta (cenozoica) sull'Antartide Orientale (Zachos et al., 2001).

La più importante conseguenza delle strette relazioni tra cambiamenti climatici e comportamento della criosfera è che le risposte dei processi glaciali e periglaciali hanno lasciato evidenti tracce sia nelle forme del rilievo conservate nelle aree deglaciate dell'Antartide, sia nei depositi superficiali del continente, sia nei sedimenti marini attorno al continente di ghiaccio, che quindi rappresentano veri e propri archivi di informazioni paleoambientali. Informazioni dirette sulla storia glaciale pleistocenica e sulla sensitività dei sistemi glaciali ai cambiamenti climatici sono peraltro fornite dalle carote di ghiaccio perforate nelle calotte glaciali (EPICA-Community-Members, 2004, 2006; Jouzel et al., 2007; Lambert et al, 2008; Masson-Delmotte et al., 2010). Questi preziosi archivi naturali forniscono informazioni fino a 800.000 anni fa anche sulla storia dell'atmosfera terrestre ma, potenzialmente, potrebbero estendersi fino a 1.5 milioni di anni fa (Fisher et al., 2013).

La lunga storia glaciale dell'Antartide è stata caratterizzata da una complessa sequenza di fasi di espansione e di contrazione delle calotte, ben riconoscibili almeno dal Miocene (Barker and Cooper, 1997; Anderson, 1999; Bart et al., 2000; Cape Roberts Science Team, 2000; McKay et al., 2009; Naish et al., 2009). In particolare, i ripetuti ispessimenti delle calotte antartiche sono stati accompagnati da espansioni sulla piattaforma continentale (Fig. 1) fino a posizioni massime simili, o addirittura più avanzate, rispetto a quelle raggiunte nell'Ultimo Massimo Glaciale -UMG- (Denton e Hughes, 1981; Denton et al., 1984, 1991; Anderson, 1999; Nakada et al., 2000; Huybrechts, 2002; Denton and Hughes, 2002; Pollard and DeConto, 2009; Bentley et al., 2016).

Tutti gli archivi paleoclimatici disponibili e le evidenze geomorfologiche e geologiche concordano nel riconoscere, durante il Pleistocene, ripetute fasi di avanzata e di ritiro delle calotte glaciali antartiche, accompagnate da variazioni del livello del mare, con abbassamenti fino a circa $125-130 \mathrm{~m}$ rispetto al livello attuale, come avvenuto nell'UMG (Fairbanks, 1989; Clark e Mix, 2002).

Queste imponenti variazioni climatiche e ambientali hanno avuto un notevole impatto sull'habitat costiero, modificando in primis la conformazione delle aree deglaciate potenzialmente colonizzabili da pinguini, foche e altre specie animali. 


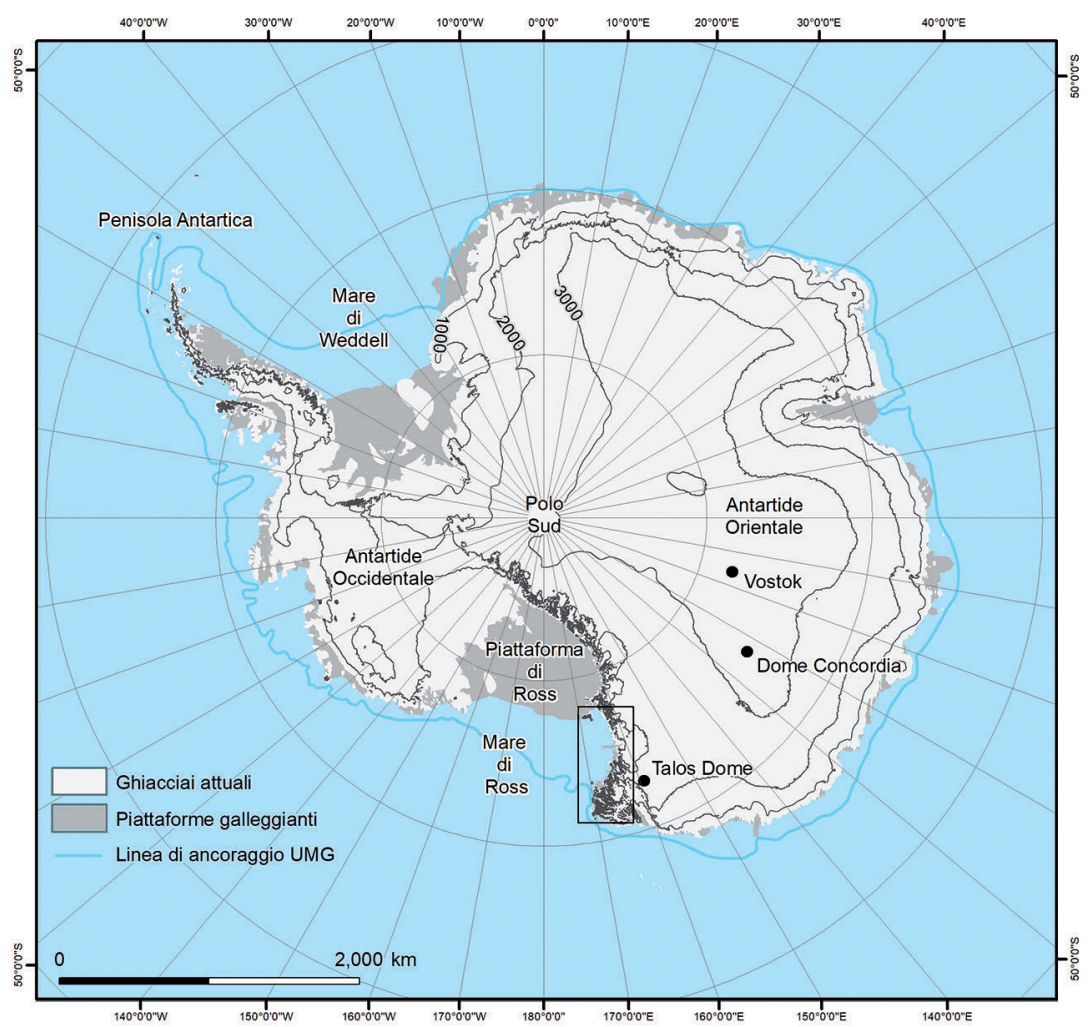

Fig. 1. L'Antartide. In grigio chiaro i ghiacciai attuali, in grigio scuro le piattaforme di gbiaccio (da Fretwell et al., 2013); in ciano la linea di galleggiamento nell'Ultimo Massimo Glaciale, intorno a 20.000 anni fa. (Da Bentley et al., 2014). Il riquadro indica l'area rappresentata nella Fig. 5. Le curve di livello derivano dall'Antartcic Digital Database, modificate.

\section{SENTINELLE BIOLOGICHE DEI CAMBIAMENTI CLIMATICI NELLE REGIONI POLARI}

Un'idea fondamentale della biologia evoluzionistica è che gli esseri viventi si adattino agli ambienti in cui vivono, ma gli ambienti sono instabili e le popolazioni, sollecitate dalle nuove condizioni che si vengono a creare, rispondono a questi cambiamenti in modo diverso (Lambert et al., 2010). In particolare, le popolazioni possono: 1) adat- 
tarsi alle nuove condizioni, 2) migrare per allontanarsi da ambienti non più idonei alla loro sopravvivenza, 3) approfittare dei nuovi babitat che si stanno sviluppando altrove, 4) regolare il numero di individui che costituisce quella popolazione, 5) cercare una diversa combinazione tra le possibilità sopra elencate.

Il modo più semplice che molte specie animali adottano per reagire ai cambiamenti climatici e ambientali, piuttosto che adattarsi alle nuove condizioni, è semplicemente quello di migrare (se possibile), per rimanere nelle condizioni di vita preferite seguendo l'ambiente ideale che si sta spostando altrove (a latitudini più alte oppure a quote più elevate). Talvolta la possibilità di migrare è preclusa dalla definitiva scomparsa dell'babitat ideale, che può avvenire in tempi troppo rapidi per consentire la migrazione in aree rifuglio (perché troppo distanti o semplicemente ignote o isolate e quindi irraggiungibili).

Durante le fasi glaciali, le specie che vivevano negli ambienti polari hanno avuto la possibilità di espandersi andando ad occupare (come ospiti freddi) ambienti posti a latidudini più basse. In realtà, i cambiamenti climatici e ambientali condizionano la biodiversità inducendo reazioni sia ecologiche sia evolutive e le risposte possono essere differenti ai diversi livelli della catena trofica, secondo meccanismi anche complessi e non sempre definibili a priori. Questo meccanismo è ben spiegato ed è chiaramente comprensibile se pensiamo alla dinamica delle specie mutualmente esclusive che si sono alternate nel corso del Pleistocene come ospiti caldi e freddi negli ambienti marini di media latitudine (ad esempio, lo Strombus bubonius e l'Arctica islandica nel Mar Mediterraneo). Un altro esempio, molto caratteristico, è il "pinguino dell'Emisfero Settentrionale" (Alca impennis Linnaeus, 1758; Sin. Pinguinus impennis) un alcide oggi estinto (Fig. 2). Seguendo le fredde correnti atlantiche, nelle fasi glaciali si spinse molto a Sud fin sulle coste della Florida lungo il margine orientale del continente americano; sul versante europeo, attraverso lo stretto di Gibilterra, l'Alca si stabilì nel Mediterraneo come ospite freddo, predato dall'uomo durante il Paleolitico medio e superiore (Ascenzi e Segre, 1971; Bonfiglio et al., 1986). Dopo l'UMG, seguendo la progressiva deglaciazione, l'Alca si ritirò nell'Atlantico settentrionale dove fu oggetto di progressiva predazione da parte dell'uomo per l'intero Olocene. L'ultima colonia sull'Isola di Funk (Canada) vide il progressivo declino già nel XVI secolo, fino all'estinzione avvenuta tra il XVIII e la prima metà del XIX secolo (Grieve, 1885; Fuller, 1999; Serjeantson, 2001). 


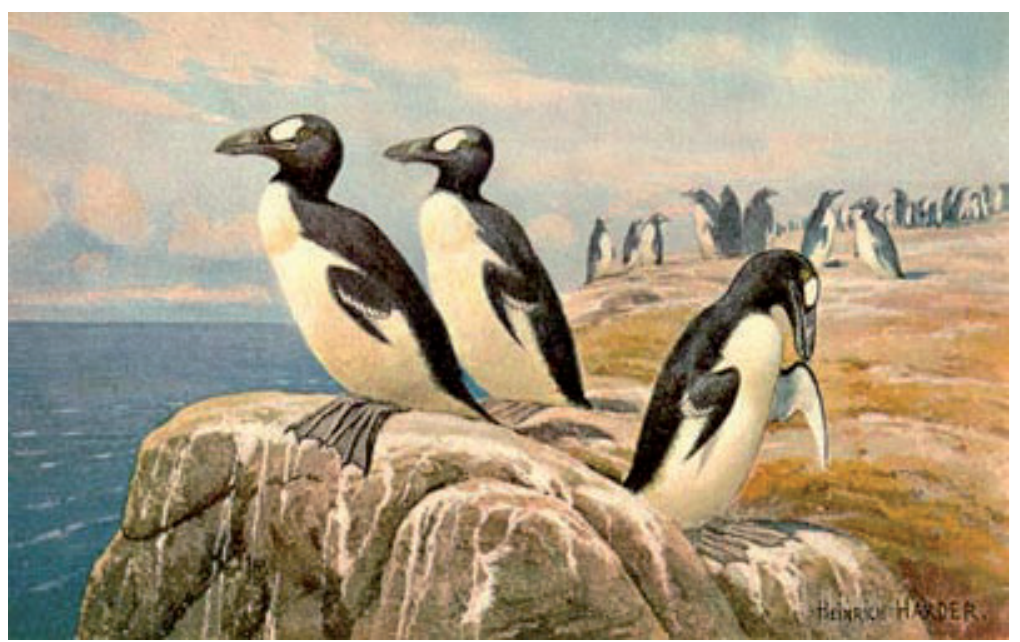

Fig. 2. Alca impennis Linn.; Sin. Pinguinus impennis un alcide estinto equivalente, nell'emisfero settentrionale, ai pinguini, ospite freddo nel Mar Mediterraneo durante le fasi glaciali. (Collector cards from Tiere der Urwelt, Animals of the Prehistoric World, Serie Ia, datato1916). Creato da Heinrich Harder.

Da quanto sopra riportato emerge l'importanza di distinguere gli effetti dei cambiamenti climatici a seconda che si consideri il passaggio da una fase interglaciale a una fase glaciale o viceversa. La questione non è di poco conto, dato che nel primo caso il passaggio avviene tramite una più lenta transizione alle nuove condizioni, con una progressione che richiede tempi relativamente lunghi. Al contrario, è ampiamente riconosciuto che la deglaciazione o, meglio, il passaggio da fasi glaciali a fasi interglaciali, avvenga in tempi molto rapidi. Pertanto, gli ecosistemi e gli habitat specifici delle regioni fredde sono più vulnerabili all'impatto dei cambiamenti climatici durante le fasi di riscaldamento rispetto alla condizione inversa che si verifica durante le fasi di raffreddamento. Un esempio noto è quello dei mammuth e di altre specie che si sono estinte nella transizione tra l'UMG e l'Olocene.

Attualmente, molte specie sono a rischio di estinzione se il futuro riscaldamento globale continuerà con questi ritmi e molte prove convincenti al riguardo sono riportate per quasi tutte le componenti degli ecosistemi marini, dalle comunità planctoniche ai grandi mammiferi (IPCC, 2007, 2013; Simmonds and Isaac, 2007; Wassmann et 
al., 2011). Il caso più drammaticamente noto è rappresentato dall'orso polare (Ursus maritimus) che, nell'Artico, è costretto ad adattarsi rapidamente alle nuove condizioni oppure dovrà necessariamente estinguersi. D'altro canto, molte specie subartiche troveranno babitat più estesi da occupare per effetto della progressiva riduzione dell'ambiente polare e la contestuale estensione di ambienti a clima più mite.

Nell'Antartico, a partire da 34 Myr fa (limite EoceneOligocene), dopo la formazione della calotta continentale e del Fronte Polare, gli organismi si sono adattati a un ambiente estremamente freddo e isolato, caratterizzato da una marcata stagionalità. In questo ambiente, mai toccato dall'uomo prima del XIX secolo, emblematico è il caso dei pinguini, che sono certamente da considerare i veri "nativi" di quel remoto continente. A parte i pinguini imperatore (Aptenodytes forsteri) e poche altre eccezioni, i pinguini si riproducono nelle aree deglaciate delle coste antartiche. Tra questi, il pinguino di Adelia (Pygoscelis adeliae, Hombron \& Jacquinot 1841) è la specie dominante (Ainley, 2002; Santora et al., 2020).

Grazie alla loro ben nota elevata sensibilità ambientale, i pinguini di Adelia rappresentano attualmente una delle specie più studiate, soprattutto per quanto riguarda la comprensione dei cambiamenti climatico-ambientali che si registrano nell'Oceano Meridionale (Taylor et al., 1990; Woeheler, 1993; Croxall et al., 2002; Ainley, 2002; Ainley et al., 2005; Forcada and Trathan, 2009; Shepherd et al., 2005; Lynch e LaRue, 2014; Borowicz et al., 2018; Santora et al., 2020).

Se dovessero realizzarsi gli scenari più drammatici previsti da un'accelerazione del riscaldamento globale, i pinguini di Adelia non avrebbero alcun luogo abbastanza freddo in cui rifugiarsi per sfuggire alle mutate condizioni ambientali e dovrebbero necessariamente adattarsi in tempi rapidi oppure sarebbero destinati all'estinzione (Lambert, 2010). Questo li rende formidabili indicatori del clima glaciale dell'Antartide, veri e propri campanelli d'allarme dei cambiamenti climatici (Ainley, 2002).

\section{I PINGUINI DI ADELIA}

I pinguini di Adelia (Pygoscelis adeliae) sono considerati la specie più resistente alle crude ed estreme condizioni antartiche. Durante 
l'estate australe, tra fine ottobre e febbraio, i pinguini nidificano per riprodursi sulle aree deglaciate delle coste antartiche, aggregandosi in colonie di numerosi individui. Letteralmente, milioni di individui nidificano in zone libere dai ghiacci lungo la costa del continente e delle isole che la costellano. Durante l'inverno, quando l'oscurità persiste 24 ore al giorno e la temperatura scende sotto i $-40{ }^{\circ} \mathrm{C}$, i pinguini vivono al margine esterno del ghiaccio marino, ben lontani dalle aree costiere dell'Antartide. Quando la luce del sole ritorna, le temperature possono essere ancora al di sotto di $-20^{\circ} \mathrm{C} \mathrm{ma}$, tra settembre e ottobre, i pinguini viaggiano per centinaia e centinaia di chilometri, camminando o scivolando sul ghiaccio marino per raggiungere le colonie sulla terraferma. Per primi arrivano i maschi che occupano e riassettano i nidi, costituiti da concentrazioni di ciottoletti centimetrici dove la femmina depone due uova (Fig. 3). Le colonie sono costituite concentrazioni di questi nidi, da alcune migliaia a diverse centinaia di migliaia di nidi (Fig. 4).

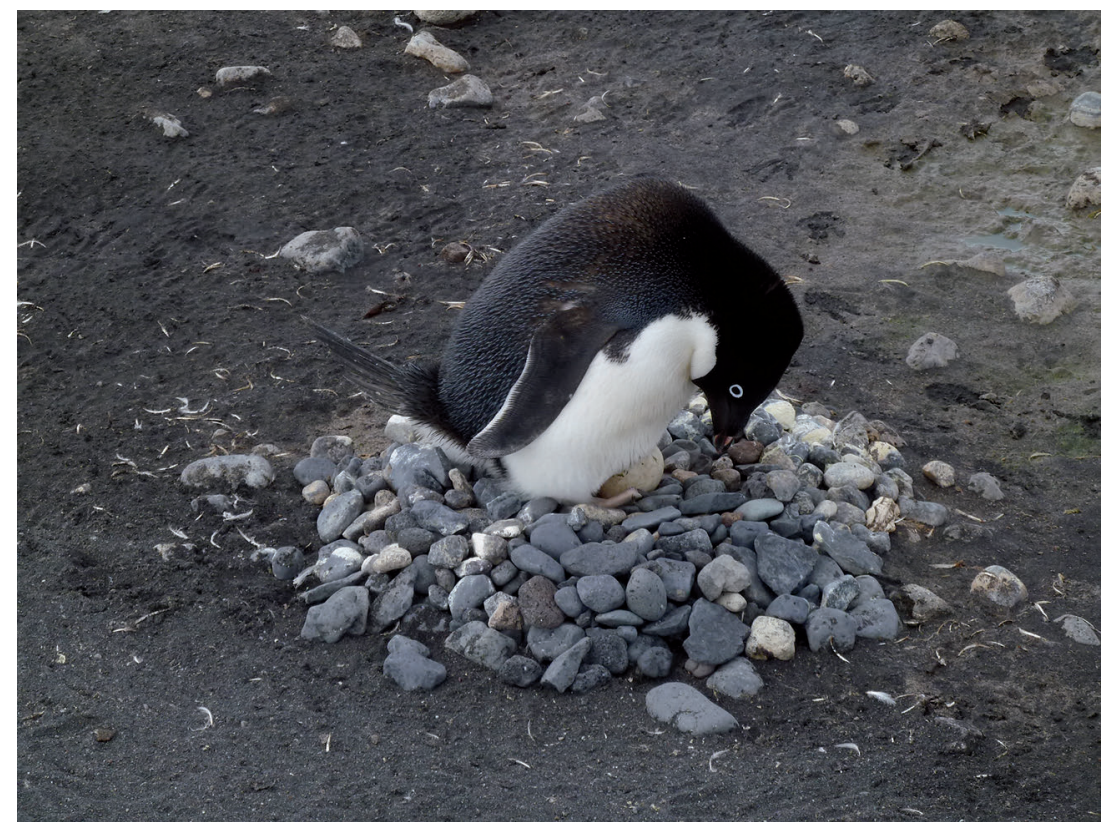

Fig. 3. Pinguino di Adelia in un nido di ciottoli con uovo appena deposto a Cape Bird, Isola di Ross, Antartide. (Foto C. Baroni, dicembre 2011, @ PNRA). 

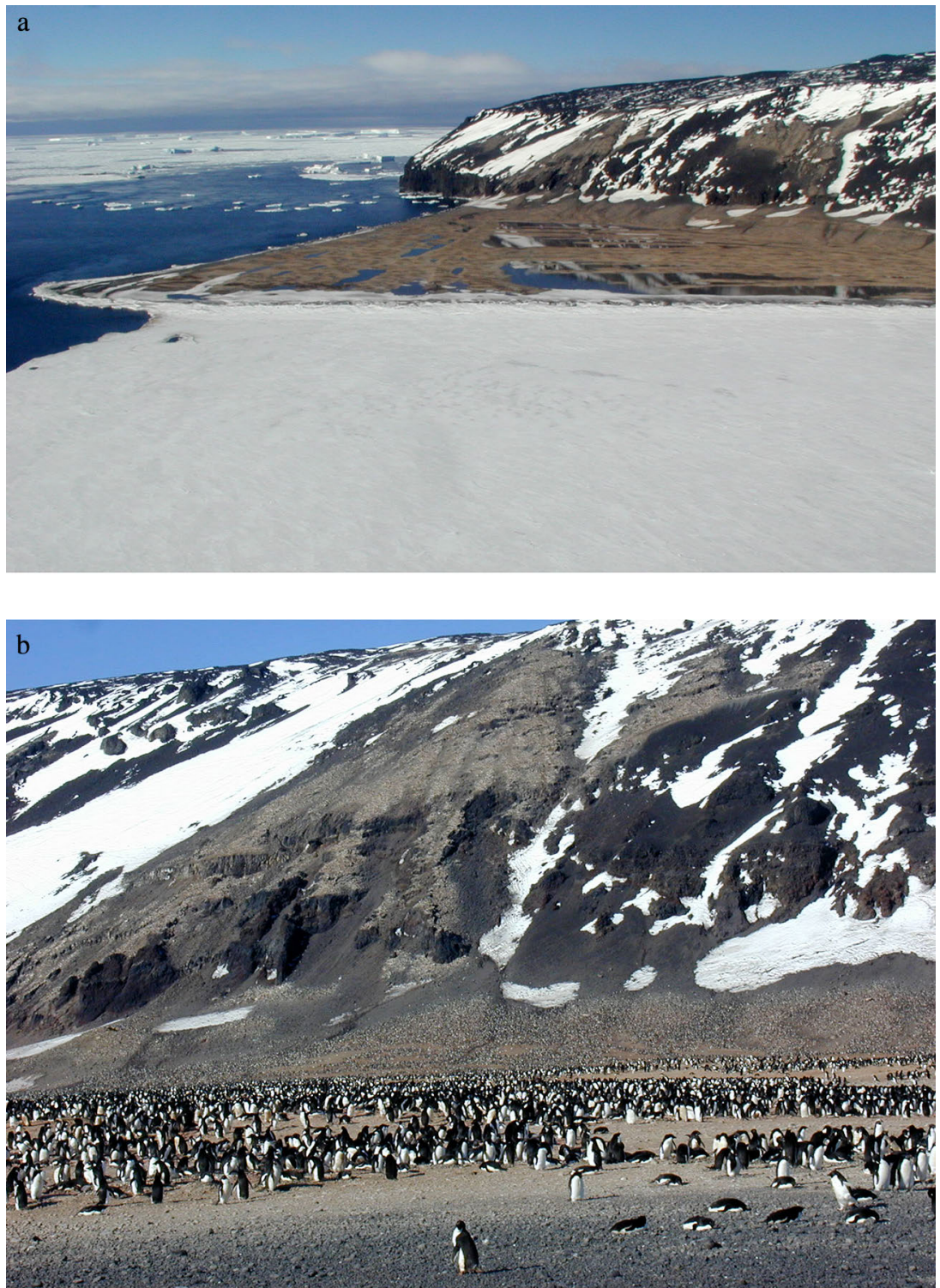

Fig. 4. a) La più estesa colonia al Mondo di pinguini di Adelia a Cape Adare, Terra Vittoria, Antartide. (Foto C. Baroni, dicembre 2002, @ PNRA).b) Nidi di pinguini di Adelia a perdita d'occhio, colonia di Cape Adare, Terra Vittoria, Antartide. (Foto C. Baroni, gennaio 2003, @ PNRA). 
Il censimento della popolazione riproduttiva degli Adelia ha rilevato 3,79 milioni di coppie (oltre 7,5 milioni di pinguini) distribuite in oltre 250 siti di riproduzione (Lynch e Larue, 2014), che corrisponderebbero a 189 colonie sensu Ainley (2002; Santora et al., 2020). Il censimento non considera $\mathrm{i}$ pinguini in età non ancora riproduttiva e, quindi, la popolazione totale di pinguini di Adelia potrebbe superare i 9-10 milioni di individui. Un terzo della popolazione adulta si trova nel Mare di Ross (33\%), il 21\% occupa la Penisola Antartica, il 30\% l'Antartide Orientale e il resto le rimanenti aree deglaciate dell'Antartico (Lynch e Larue, 2014). Nel Mare di Ross si trovano sia la più estesa colonia del mondo, a Cape Adare, che ospita 338.000 coppie (Lynch e Larue, 2014), sia la colonia più meridionale del nostro Pianeta, a Capo Royds ( $77^{\circ} 30^{\prime} \mathrm{S}$ ), nell'Isola di Ross (Fig. 5).

I pinguini di Adelia tornano istintivamente nelle stesse colonie dove sono stati generati, mostrando quindi una elevata filopatria natale; in passato, si pensava che esistessero bassi livelli di migrazione tra le diverse colonie, il che, in realtà, è abbastanza vero in periodi di condizioni ambientali e climatiche relativamente stabili (Ainley, 2002; Shepherd et al., 2005). D'altra parte, una forte filopatria natale dovrebbe promuovere la divergenza genetica tra le popolazioni. Tuttavia, stanno emergendo prove di un elevato livello di flusso genico episodico tra le colonie di pinguini, molto probabilmente causato dalla migrazione forzata da condizioni ambientali che impediscono l'accesso ad alcune colonie (Shepherd et al., 2005). Emblematico è il caso dei mega-iceberg distaccati dalla Piattaforma di Ross, che si sono arenati recentemente impedendo l'accesso ad alcune colonie dell'Isola di Ross. Lo sbarramento delle normali rotte migratorie dei pinguini ha avuto drammatiche conseguenze non solo sull'occupazione dei siti di riproduzione ma anche sul foraggiamento e, in alcuni casi, ha pesantemente influito addirittura sul successo riproduttivo.

I principali fattori ecologici che regolano e limitano la distribuzione dei pinguini di Adelia intorno all'Antartide sono: 1) le aree deglaciate lungo i margini costieri, 2) l'estensione e la persistenza della banchisa, 3) la presenza di acque libere dal ghiaccio marino nei pressi della colonia per consentire l'accesso al mare (foraggiamento), 4) la disponibilità di cibo (krill, piccoli pesci e calamari), 5) la relativa assenza di predatori. 


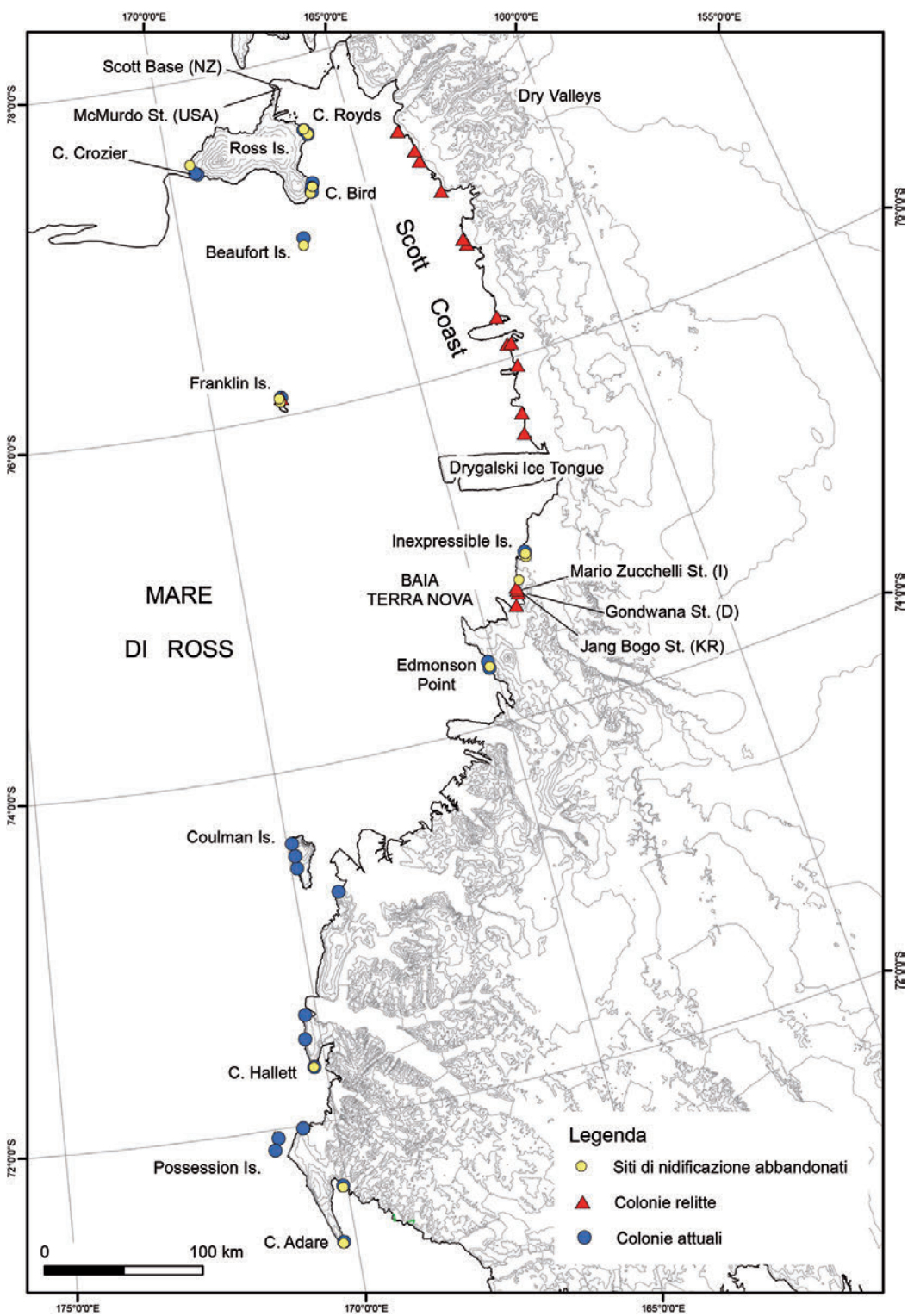

Fig. 5. La Terra Vittoria, Antartide. Distribuzione delle attuali colonie di pinguini di Adelia (pallini blu), dei siti di nidificazione abbandonati ai margini delle colonie attualmente occupate (pallini gialli) e delle colonie relitte (triangoli rossi). [Dati dall'Antarctic Digital Database - ADD (https://www.scar.org/data-products/antarctic-digital-database/)]. 
I pinguini stanno attualmente reagendo ai cambiamenti climatici spostando le loro colonie riproduttive a latitudini più meridionali. Uno dei più evidenti segnali del riscaldamento in atto viene dalla Penisola Antartica, dove la temperatura media invernale dell'aria è aumentata più rapidamente $\left(6{ }^{\circ} \mathrm{C}\right.$ dal 1950$)$ che in qualsiasi altra parte del mondo (Stokstad, 2007). Nuovi dati sul censimento dei pinguini di Adelia mostrano che la popolazione della Penisola si sarebbe notevolmente ridotta rispetto al 1993 e almeno otto colonie sarebbero state abbandonate (Lynch e LaRue, 2014). Contestualmente, la popolazione del Mare di Ross e dell'intera Antartide Orientale è aumentata sia in termini di numero di individui $(53 \%)$ sia in termini di nuove colonie (almeno 11 nuove colonie). Allo stesso tempo, altre specie di pinguini hanno occupato le nicchie ecologiche abbandonate dagli Adelia nella Penisola Antartica (Forcada e Trathan, 2009).

\section{IL PATRIMONIO DI INFORMAZIONI CUSTODITO NELLE COLONIE DEI PINGUINI DI ADELIA}

\subsection{Colonie di pinguini e suoli ornitogenici}

Le attuali colonie di pinguini di Adelia si sviluppano sulle spiagge emerse oloceniche e sui terrazzi marini, ma si espandono anche sui coni e sulle falde detritiche, sui rock glaciers pedemontani, sulle morene deposte e/o a nucleo di ghiaccio delle aree costiere. In alcune colonie i pinguini possono arrampicarsi per nidificare sui versanti in roccia spingendosi fino a qualche centinaio di metri di quota (Fig. 4). L'estensione delle colonie varia in funzione della popolazione di pinguini e può estendersi nell'entroterra costiero da diverse centinaia di metri fino ad alcuni chilometri. Ogni anno, i genitori dei pinguini sistemano i sassolini selezionati con il becco per costruire il nido (Fig. 6). In una colonia si osservano aggregazioni di nidi che possono variare da alcune migliaia a diverse centinaia di migliaia di unità (Fig. 4). Durante la stagione riproduttiva, la predazione di uova e di pulcini da parte degli skua antartici (Catharacta maccormicki; Fig. 7), lascia al suolo una gran quantità di resti di pinguino (gusci d'uovo, carcasse di pulcini, ossa, piume, frammenti di pelle e altri resti organici), che si mescolano alle deiezioni (guano) e ai ciottoletti dei nidi. Con il trascorrere del tempo, il guano, i nidi e i resti della predazione da parte degli skua si sovrappongono e si saldano tra loro accumulandosi a 
formare veri e propri suoli organici, riconosciuti fin dalle spedizioni antartiche del XIX secolo (Fig. 8). Henryk Arctowski, geologo della Spedizione Antartica Belga al comando di de Gerlache (1898-99), raccolse in depositi di guano i primi insetti mai trovati in Antartide.

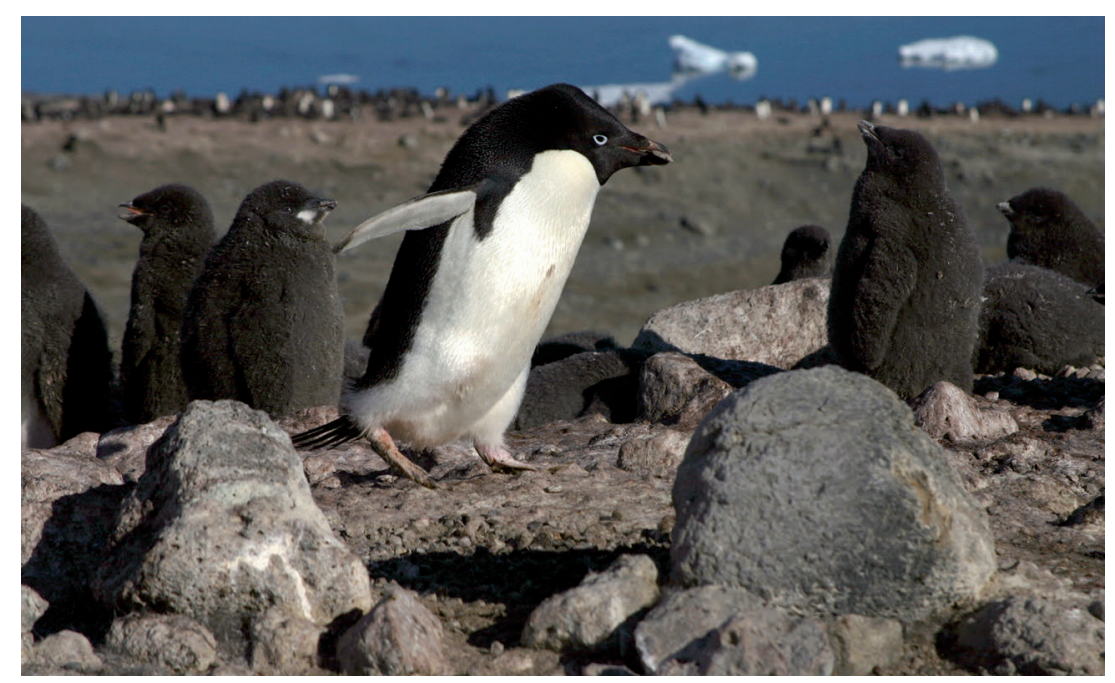

Fig. 6. Pinguino di Adelia che trasporta col becco un ciottolo per assemblare il nido, colonia di Cape Bird, Isola di Ross, Antartide. (Foto C. Baroni, gennaio 2007, @ PNRA).

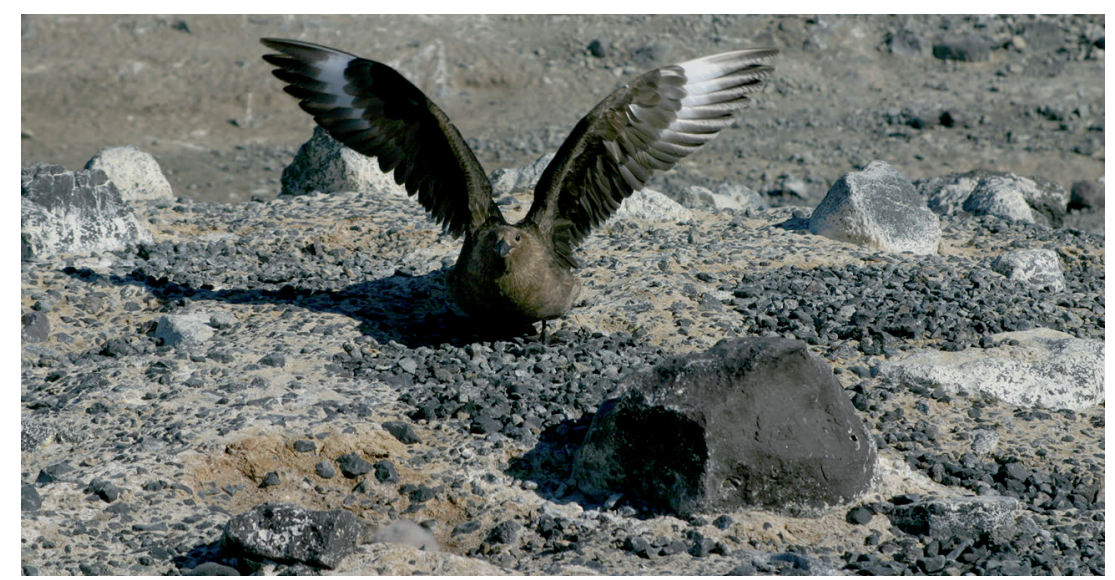

Fig. 7. Skua antartico, il principale predatore delle colonie di pinguini di Adelia, Cape Bird, Isola di Ross, Antartide. (Foto C. Baroni, gennaio 2007, @ PNRA). 


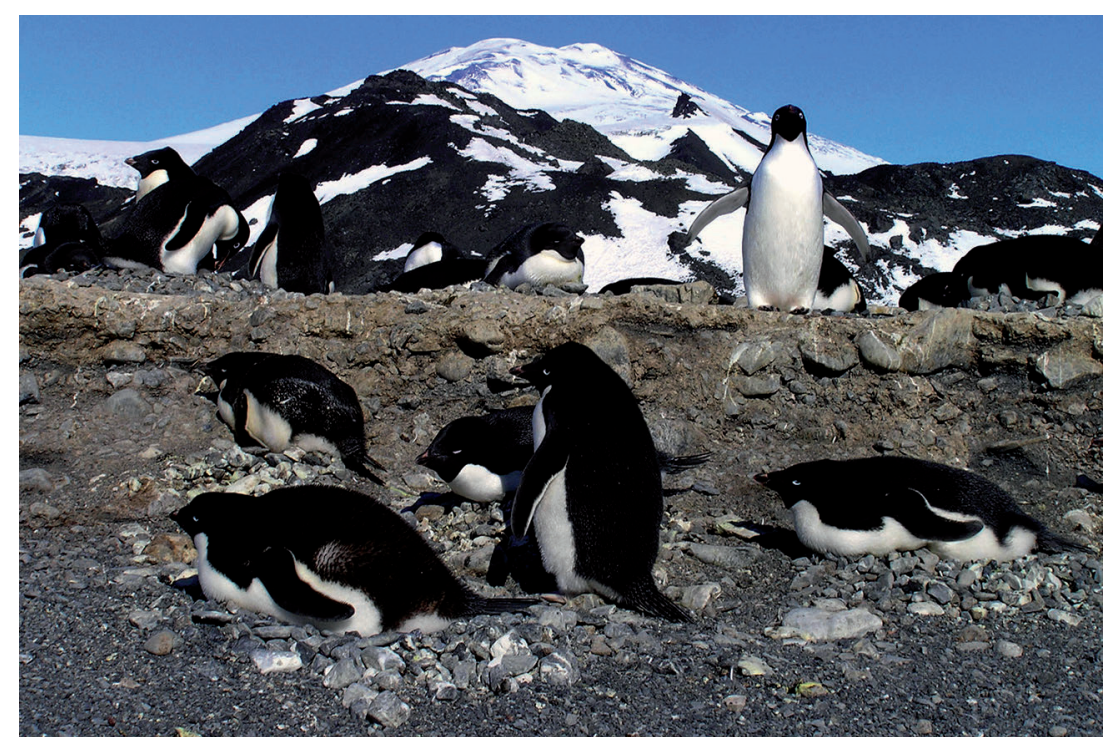

Fig. 8. Nidi di pinguini di Adelia, Edmonson Point, Terra Vittoria, Antartide. Si noti il suolo ornitogenico visibile al centro della foto, creato dall'accumulo di deiezioni (guano) resti organici e nidi sovrapposti. (Foto C. Baroni, dicembre 2006, @ PNRA).

I terreni organici delle colonie di pinguini furono definiti suoli ornitogenici da Syroechkovsky (1959) e sono stati descritti per la prima volta nella Terra Vittoria (Harrington, 1960; Ugolini, 1972; Campbell e Claridge, 1987). Questi suoli costituiscono un eccellente archivio di informazioni sui processi pedogenetici e sulla chimica dei suoli organici che si sviluppano in condizioni ambientali veramente estreme (Ugolini, 1972; Speir e Cowling, 1984; Campbell e Claridge, 1987; Heine e Speir, 1989; Tatur e Myrcha, 1989; Zhu et al, 2005; Michel et al., 2006; Simas et al., 2007, 2008).

In una colonia occupata da lungo tempo il guano si accumula creando orizzonti di diversi decimetri di spessore: tanto maggiore è la durata dell'occupazione quanto maggiore sarà lo spessore dei suoli (Ugolini, 1972). Una duratura fase di occupazione delle colonie si riflette in un progessivo accrescimento dei depositi organici; inoltre, i nidi, le carcasse e gli altri resti organici possono eventualmente essere coperti da depositi di versante o di altra natura. Tipici sono i depositi colluviali trasportati dalle acque di fusione nivale che localmente possono intercalarsi ai suoli organici. 
Alla periferia o nelle vicinanze delle colonie attualmente occupate si trovano siti di nidificazione abbandonati, che testimoniano sia la persistenza nel tempo delle condizioni favorevoli alla colonizzazione, sia la variabilità del numero di pinguini che costituiscolo le popolazioni che occupano quelle colonie (Fig. 9). Questi siti sono stati riconosciuti anche a chilometri di distanza dal centro delle colonie attuali (Baroni e Salvatore, dati inediti). Inoltre, siti di nidificazione abbandonati sono stati individuati anche in aree oggi non più occupate da colonie di pinguini (Baroni e Orombelli, 1994; Fig 5). Si tratta di vere e proprie colonie relitte che custodiscono preziose informazioni sulla storia dei pinguini e dell'ambiente in cui hanno vissuto.

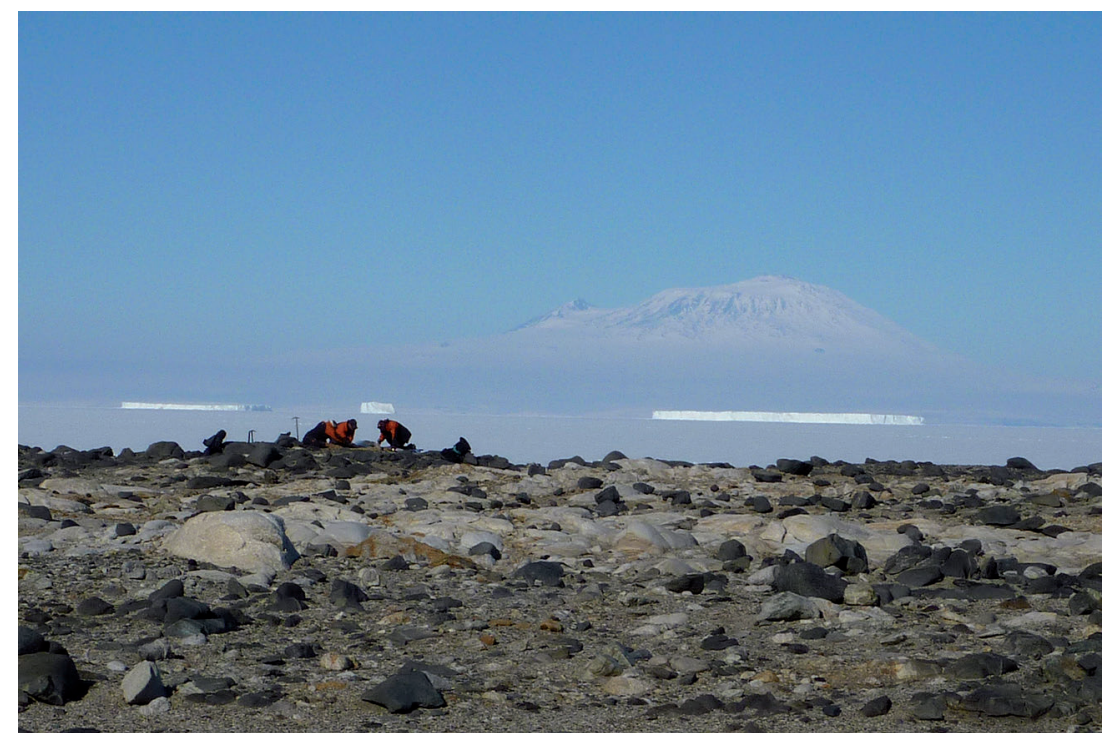

Fig. 9. Scavo di una colonia relitta di pinguini di Adelia, Scott Coast, Terra Vittoria, Antartide. (Foto C. Baroni, gennaio 2013 @ PNRA).

Dopo l'abbandono dei siti di nidificazione, i forti venti antartici hanno spazzato la superficie di occupazione asportando le componenti più superficiali e più fini, generando veri e propri pavimenti eolici di deflazione costituiti da plaghe di ciottoletti concentrate in corrispondenza dei vecchi nidi, che raggiungono anche estensioni di decine/centinaia di metri quadrati. Una diffusa copertura di licheni ammanta i ciottoli delle più antiche colonie relitte. 
Sia le colonie relitte sia i suoli ornitogenici ci forniscono un importante archivio di informazioni sulle popolazioni di pinguini di Adelia che le hanno occupate nel tempo. Per prima cosa il guano e gli altri resti di pinguino ci permettono di ottenere dati cronologici $\left({ }^{14} \mathrm{C}\right.$; Fig. 10) che scandiscono la storia della colonizzazione delle aree costiere, le fasi di ritiro delle calotte antartiche dopo l'Ultimo Massimo Glaciale e contribuiscono alla ricostruzione delle curve di sollevamento relativo delle aree costiere (Baroni e Orombelli, 1991; Baroni, 1984, 2013; Emslie, 1995; Emslie et al., 1998, 2007; Baroni e Hall, 2004; Hall et al., 2004). Se consideriamo le colonie di pinguini "sentinelle" dei recenti cambiamenti climatici (Ainley, 2002), i dati ottenuti dalla distribuzione delle colonie relitte e dai siti di nidificazione abbandonati in colonie ancora occupate ci forniscono informazioni rilevanti sulle risposte ecologiche dei pinguini agli episodi dei passati cambiamenti ambientali e climatici.

Inoltre, l'ambiente antartico, freddo e secco, conserva nei suoli ornitogenici carcasse di pinguini e altri resti (ossa, pelle, piume, guano e gusci d'uovo) che hanno fornito il DNA antico (sia nucleare che mitocondriale) meglio conservato e mai scoperto (Lambert et al., 2002; Ritchie et al., 2004, Millar et al., 2008; Subramanian et al., 2009; Parks et al. 2015).

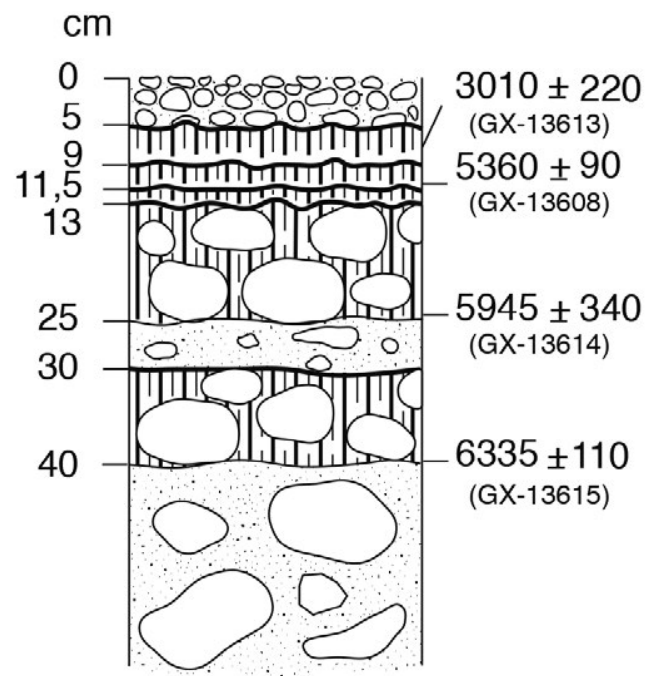

Fig. 10. Inexpressible Island (Fig. 5), sovrapposizione di diversi livelli di guano (linee vericali) generati da fasi di occupazione sovrapposte che hanno restituito diverse date ${ }^{14} \mathrm{C}$ (numeri sulla destra; tra parentesi le sigle dei campioni datati. (Ridisegnato da Baroni e Orombelli, 1991). 
Le sequenze di DNA estratte dalle ossa di pinguino di Adelia hanno permesso di definire variazioni genetiche e trend evolutivi indotti dai cambiamenti climatico-ambientali che hanno accompagnato le fasi glaciali e interglaciali (tipicamente le fasi di raffreddamento climatico che hanno accompagnato le glaciazioni e le fasi di riscaldamento registrate negli interglaciali e nell'Olocene).

\subsection{La deglaciazione e la ri-colonizzazione delle aree costiere}

Durante il Pleistocene, le ripetute fasi di espansione e di collasso delle calotte antartiche hanno impattato sull'babitat dei pinguini a scala continentale, allontanandoli dalle numerose ma discontinue aree deglaciate distribuite lungo le coste (Fig. 1). Durante l'ultimo periodo di massima espansione glaciale (UMG, tra 25.000 e 18.000 anni fa), le aree costiere antartiche erano in gran parte coperte da ghiaccio, in seguito all'ispessimento dei margini delle calotte e alla loro avanzata su ampi settori della piattaforma continentale. In conseguenza dell'abbassamento del livello del mare a scala globale (circa -120/130 m rispetto all'attuale livello) la linea di ancoraggio delle piattaforme è avanzata di centinaia di chilometri spingendo molto più a Nord, rispetto alla posizione attuale, sia il margine delle piattaforme galleggiani, sia il limite del ghiaccio marino (Dawson, 1992; Bentley et al., 2016). Lo spessore dei ghiacciai raggiungeva, sul margine costiero, diverse centinaia metri, innalzandosi localmente fino a 400-500 $\mathrm{m}$ al di sopra del livello del mare attuale (come documentato dai depositi glaciali e dalla distribuzione dei massi erratici; Denton e Hughes, 1981, 2002; Orombelli et al., 1990; Denton et al., 1991).

L'ultima transizione dal periodo glaciale all'attuale interglaciale (Olocene) ha indotto un innalzamento della temperatura sull'Antartide di circa $12{ }^{\circ} \mathrm{C}$ che si è verificato molto repentinamente. In pochi millenni le calotte antartiche si sono ritirate nella loro posizione attuale, insieme alle piattaforme galleggianti e alla loro linea di ancoraggio. Di conseguenza, la deglaciazione è stata accompagnata dall'ingressione marina e dal sollevamento glacio-isostatico delle zone costiere. Questi eventi hanno portato alla formazione di spiagge emerse oloceniche.

Molto significativo è il caso di studio del Mare di Ross e, in particolare, della colonizzazione della Terra Vittoria, dove i pinguini di Adelia occupano un ecosistema marino molto particolare, tra i più meridionali del Pianeta. Attualmente, circa 1/3 della popolazione totale 
di pinguini di Adelia occcupa il margine occidentale del Mare di Ross, distribuita in 20 colonie lungo il margine costiero della Terra Vittoria (Fig. 5).

Tamite l'analisi di fotografie aeree e di immagini da satellite, rilevamenti geomorfologici regionali e studi geomorfologici di dettaglio condotti in siti chiave sono state studiate le aree costiere dell'intera Terra Vittoria. L'accurato rilevamento geomorfologico regionale per l'analisi del paesaggio delle aree deglaciate (Landscape analysis), affiancato da un approccio tipicamente "archeologico" per il ritrovamento dei siti di colonizzazione ("survey" areale) ci ha permesso di scoprire numerose colonie di pinguini relitte tra Cape Adare e l'Isola di Ross (Baroni e Orombelli, 1991, 1994; Baroni, 1994; Lambert et al., 2002; Baroni e Hall, 2004; Hall et al., 2004; Ritchie et al., 2004; Baroni e Salvatore, dati inediti; Fig. 5).

Applicando le tecniche proprie dell'archeologia, attraverso scavi stratigrafici dei siti di nidificazione abbandonati, è stato possibile identificare le fasi occupazionali delle colonie (eventualmente separate da strati minerali come sabbia e ghiaia di origine colluviale o periglaciale, depositi eolici, ecc.) e ricostruire la storia della colonizzazione costiera. Sono stati eseguiti saggi di scavo (generalmente $1-2 \mathrm{~m}^{2}$ ) o veri e propri scavi estesi alcuni $\mathrm{m}^{2}$, pulendo la superficie dall'alto e scavando livello dopo livello i suoli organici, fino ai sedimenti sterili sottostanti oppure fino al substrato roccioso. Inoltre, con un accurato approccio stratigrafico, è stato possibile discriminare, già durante il lavoro di scavo, tra i resti appartenenti a singoli individui e quelli attribuibili a organismi distinti, consentendo anche di riconoscere e caratterizzare i livelli organici eventualmente rimaneggiati da processi periglaciali o di altra natura. La elevata concentrazione di resti organici ha fornito numerose date ${ }^{14} \mathrm{C}$ (circa 200) che ci consentono di scandire con buona approssimazione le fasi della deglaciazione, l'arretramento della linea di ancoraggio delle piattaforme galleggianti e i tempi dell'ingressione marina nell'Olocene.

Nel Mare di Ross, la regressione glaciale si è completata intorno a circa 8000 anni fa, che all'incirca corrisponde all'età della più antica spiaggia emersa olocenica che si trova circa $30 \mathrm{~m}$ al di sopra del livello del mare attuale e all'età delle più antiche colonie di pinguini occupate dopo la deglaciazione (Baroni e Orombelli, 1991, 1994; Baroni e Hall, 2004). In realtà, il ritiro della linea di ancoraggio delle piattaforme galleggianti è stata un poco più articolata ed è avvenuta in fasi distinte tra circa 8000 e circa 3000 anni fa (Convay et al., 1999; Hall et al., 2004). 
Il sollevamento delle aree costiere è ben descritto da curve di innalzamento relativo del livello del mare basate su numerose date ${ }^{14} \mathrm{C}$ ottenute da conchiglie raccolte nei sedimenti marini (che hanno fornito età contemporanee e/o età massime per il livello del mare nel corso dell'Olocene) e da resti organici provenienti da suoli ornitogenici di colonie relitte e siti abbandonati di nidificazione individuati sulle spiagge oloceniche (che hanno fornito età minime; Baroni e Orombelli, 1991; Baroni e Hall, 2004; Hall et al., 2004). Nella zona di Baia Terra Nova, il tasso di sollevamento iniziale nei primi millenni dopo la deglaciazione è stato di $8-10 \mathrm{~mm} /$ anno, ridotto a meno di $2 \mathrm{~mm} / \mathrm{a}$ negli ultimi tre millenni $(<0.5 \mathrm{~mm} /$ a nell'ultimo millennio).

\subsection{Le paleodiete dei pinguini}

L'eccellente conservazione della sostanza organica nell'ambiente secco e freddo dell'Antartide permette anche la conservazione dei resti delle specie predate dai pinguini, concentrate nelle deiezioni trattenute nei suoli ornitogenici. Ciò consente, pertanto, anche di studiare i resti delle paleodiete dei pinguini e di ricostruire le strategie di foraggiamento. Ossa, otoliti e altri resti di pesci, oltre a becchi di calamaro (chitinosi), sono perfettamente conservati nei suoli e hanno fornito rilevanti informazioni sulle paleodiete dei pinguini di Adelia. L'identificazione tassonomica del "silverfish" antartico (Pleuragramma antarcticum) e dei calamari glaciali (Psychroteuthis glacialis) ha rivelato queste due specie come importanti componenti nella dieta del pinguino di Adelia fin dall'inizio dell'Olocene (Emslie et al., 1998; Emslie, 2001; McDaniel and Emslie, 2002; Emslie and McDaniel, 2002; Polito et al., 2002; Emslie and Woehler, 2005; Lorenzini et al., 2009).

La proporzione di questi resti nella paleodieta dei pinguini è variata in accordo con le modificazioni climatico ambientali che hanno interessato le coste antartiche e, in particolare, il settore del Mare di Ross. E' noto, infatti, che le strategie di foraggiamento degli Adelia sono legate alle condizioni ambientali locali e regionali e i dati recentemente acquisiti nella Terra Vittoria sembrano confermare che questo sia avvenuto anche nel passato. Pertanto, la variabilità delle specie predate dai pinguini rappresenta un valido strumento per studiare, da un altro punto di vista, gli effetti indotti dai cambiamenti globali sugli babitat dei pinguini (Ainley et al., 1998; Ainley, 2002).

I campioni di suolo raccolti nelle colonie della Terra Vittoria 
hanno fornito migliaia di resti di pesce (vertebre, ossa, otoliti, ...) e di altri organismi predati dai pinguini. Gli otoliti raccolti sono stati identificati sulla base di tavole anatomiche comparative dei diversi taxa antartici (Williams e McEldowney, 1990). Lidentificazione tassonomica degli otoliti ha permesso di dimostrare che la specie principalmente predata dai pinguini è il Pleuragramma antarcticum Boulenger 1902, noto come "silverfish" ( $88 \%$ del numero totale di otoliti) e, in ordine decrescente d'importanza, il Trematomus bernacchii Boulenger 1902 $(1,1 \%)$ e il Trematomus scottii Boulenger 1907 (0,13\%); un certo numero di otoliti è stato identificato anche come Trematomus sp. $(0.3 \%)$ e Pagothenia sp. $(1.4 \%)$.

Inoltre, analisi quantitative condotte su circa 400 otoliti hanno consentito di definire anche le dimensioni medie della taglia dei pesci preferibilmente predati, compresa tra 40 e $80 \mathrm{~mm}$ nell' $83 \%$ dei casi (67 $\pm 23 \mathrm{~mm}$; Lorenzini et al., 2009).

Numerosi campioni di guano non contengono resti di specie predate, riflettendo presumibilmente diete a base di krill, i gamberetti antartici che non restano conservati nei suoli, ma che sono eventualmente individuabili attraverso l'analisi degli isotopi stabili estratti dal guano di pinguino e dai gusci d'uovo. Queste analisi hanno infatti consentito di individuare anche il contributo dietetico di altre prede, tra le quali principalmente il krill (Euphausia superba), non osservato direttamente nei suoli nella forma di resti macroscopici (Lorenzini et al., 2010, 2012).

\section{GLI ELEFANTI MARINI E LA COLONIZZAZIONE DELLE COSTE ANTARTICHE}

Avvistamenti di elefanti marini meridionali (Mirounga leonina) sulle coste antartiche sono stati segnalati sin dall'epoca eroica dell'esplorazione antartica e avvengono tuttora anche alle latitudini più meridionali, lungo la costa della Terra Vittoria, ma questi mammiferi marini non sono annoverati tra gli elementi più comuni dell'ecosistema antartico (Le Boef e Laws, 1994; Biew et al., 2007). Gli elefanti marini meridionali sono, infatti, mammiferi molto mobili, ma principalmente subantartici, che prediligono acque stagionalmente libere dai ghiacci marini. Nel 1990 la popolazione totale era di 664.000 individui; oggi si stima che siano diminuiti ma non ci sono dati su censi- 
menti recenti dell'intera popolazione. Colonie riproduttive con un consistente numero di individui si trovano nella Penisola di Valdes, in Sud America, nella Georgia del Sud (circa il 60\% della popolazione totale), sulle isole subantartiche (Kerguelen, Heard e altre minori, circa il 28\% della popolazione totale) sulle isole Macquarie, Campbell, Auckland e sulle isole Antipodi (circa il 12\%). La maggior parte delle colonie si trovano a cavallo (oppure all'esterno) della convergenza Antartica e solo poche piccole colonie sono segnalate entro questo limite (De Bruyn et al,, 2009). Tra queste, le uniche colonie presenti sulle coste deglaciate dell'Antartide si trovano alla Statione Palmer sulla Penisola Antartica e sulle Isole Windmill nella Terra di Wilkes (Antartide orientale).

Nella famiglia dei focidi, gli elefanti marini, pur essendo caratterizzati da un forte dimorfismo sessuale, sono la specie che raggiunge le più grandi dimensioni. I maschi adulti possono raggiungere $4.5 \mathrm{~m}$ di lunghezza e fino a $3.7 \mathrm{t}$ di peso, mentre le femmine restano al di sotto di $2.8 \mathrm{~m}$ di lunghezza e $800 \mathrm{~kg}$ di peso (Le Boeuf and Laws, 1994).

Gli elefanti marini si alimentano in acque aperte, in assenza di banchisa, dove si muovono ad ampio raggio (migliaia di $\mathrm{km}$ ) coprendo aree di foraggiamento molto estese.

Sono grandi predatori di calamari e pesci, in grado di raggiungere la sorprendente profondità di $1430 \mathrm{~m}$. La loro impressionante capacità di immersione può durare per un tempo incredibilmente lungo, fino a 120 minuti (Le Boeuf and Laws, 1994).

Gli elefanti marini si riposano sulla terraferma, rotolandosi sulle spiagge sabbiose o ghiaiose, libere dai ghiacci, per la muta e per riprodursi. Il goffo e pesante movimento degli elefanti marini a terra ha l'effetto di bulldozer che arano le spiagge, travolgono e schiacciano qualsiasi cosa si trovi sul loro cammino. Colonie di pinguini eventualmente occupate da questi enormi mammiferi subiscono gravi danni e raramente si contano superstiti.

Durante i secoli XIX e XX questi mammiferi sono stati cacciati per la produzione di olio e la popolazione era stata pesantemente predata, giungendo quasi sull'orlo dell'estinzione (Le Boeuf eLaw, 1994). Attualmente, la popolazione sembra sottoposta a una forte riduzione, con estinzione delle colonie più settentrionali e conseguente migrazione degli elefanti marini a latitudini più meridionali, sempre più in direzione dei mari antartici (Hindel et al., 2017; Jones et al., 2020). 
Hall et al. (2006) hanno scoperto che gli elefanti marini hanno in passato occupato la Terra Vittoria, colonizzandola sin da 7000 anni fa (Fig. 11). Sulle spiagge sabbioso-ghiaiose e ciottolose presenti lungo la costa deglaciata della Terra Vittoria sono stati raccolti numerosi campioni di pelle, ossa e resti di scheletri mummificati, inclusi neonati, a dimostrazione che molti dei siti occupati lungo la Terra Vittoria non erano semplici siti occupati per la muta o come semplici aree di riposo, ma erano vere e proprie colonie riproduttive (Hall et al., 2006: Koch et al., 2019). Attualmente, a parte la polynia di Inexpressible Island nella zona di Baia Terra Nova, la Scott Coast è sbarrata da una banchisa costiera, localmente caratterizzata da ghiaccio pluriennale, e non è pertanto raggiungibile dagli elefanti marini. La colonia più vicina attualmente si trova sull'Isola Macquarie (543ㅇ' S), $2500 \mathrm{~km}$ a Nord, circa a mezza strata tra l'Antartide e l'Australia.

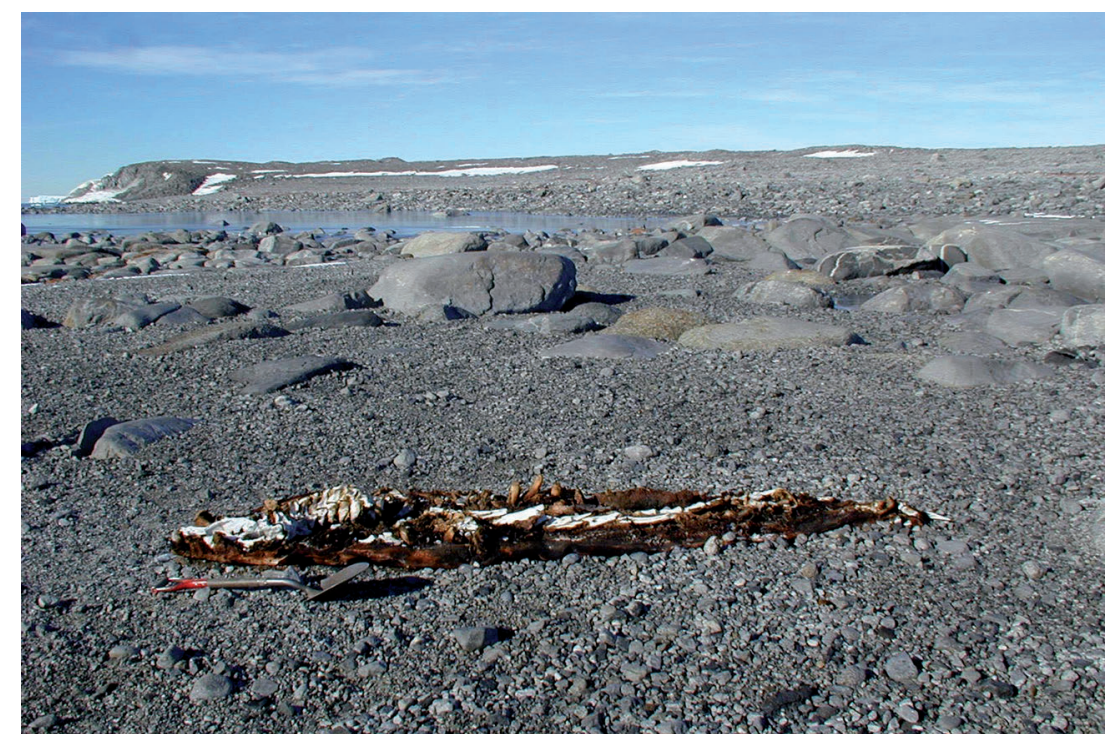

Fig. 11. Un elefante marino mummificato sulle spiagge di Inespressible Island, Baia Terra Nova, Antartide. (Foto C. Baroni, gennaio 2002, @ PNRA).

Oltre 200 campioni di resti di elefanti marini sono stati datati con il ${ }^{14} \mathrm{C}$ e utilizzati per estrarre il DNA antico, applicando stretti protocolli per sequenziare e amplificare il DNA mitocondriale (mtDNA), al fine di ricostruire la storia evolutiva della popolazione ancestrale di elefanti 
marini nel Mare di Ross (De Bruyn et al., 2009, 2014). Questa popolazione aveva stretti legami genetici con la popolazione che attualmente occupa la colonia dell'Isola Macquarie e si è insediata a partire da circa 7500 anni fa, subito dopo la deglaciazione che ha seguito l'UMG, più o meno contemporaneamente (o poco dopo) l'arrivo dei primi pinguini di Adelia. Il collasso di queste colonie è iniziato intorno a 1000 anni fa e si è completato circa 400 anni fa, in un periodo che, nell'emisfero settentrionale, corrisponde alla "piccola età glaciale".

\section{LA RISPOSTA DEI PINGUINI DI ADELIA E DEGLI ELEFANTI MARINI AI CAMBIAMENTI CLIMATICI}

Le condizioni ambientali dell'UMG hanno obbligato i pinguini e tutti gli animali che nidificano nelle aree costiere a migrare verso Nord, nel settore circum-antartico, in cerca di luoghi adatti per la nidificazione e la riproduzione. A riprova di questo comportamento dei pinguini sono emerse recentemente molte evidenze di occupazioni di colonie precedenti all'UMG, che vanno dalla scoperta di suoli ornitogenici (sepolti da depositi glaciali dell'UMG), a resti di scheletri subfossili di pinguini, ad altri resti organici associati alle colonie (Ritchie et al 2004; Gardner et al., 2006; Subramanian et al., 2009; Emslie et al., 2007; Parks et al., 2015). Dopo la deglaciazione, le zone costiere libere dal ghiaccio erano perfettamente disponibili per essere ri-colonizzate. I pinguini di Adelia hanno riconquistato i siti ancestrali colonizzati dai loro progenitori del Pleistocene.

Il tasso evolutivo calcolato dal DNA antico prelevato dai resti fossili di pinguini, rispetto agli eventi di divergenza osservati nel Pleistocene, ha permesso a Ritchie et al. (2004) di riconoscere due popolazioni con un distinto patrimonio genetico (lineage) nel DNA mitocondriale. Un primo "lineage" è stato osservato principalmente nelle colonie del Mare di Ross, mentre un secondo è stato evidenziato nella popolazione circumantartica. La diversa distribuzione dei due patrimoni genetici è coerente con l'esistenza di due rifugi ancestrali (pleistocenici) che erano soggetti a strozzature nelle dimensioni della popolazione (bottleneck). Il tasso di evoluzione molecolare della regione HVRl, determinato estraendo il DNA mitocondriale da 162 ossa subfossili che abbracciano un arco temporale di circa 37.000 anni, era di 0,96 sostituzioni per sito ogni milione di anni (Lambert et al., 2002). 
Applicando questo tasso evolutivo, il più recente progenitore di questi pinguini avrebbe un'età compresa tra 75.000 e 122.000 anni, certamente più antico dell'UMG, possibilmente più antico dell'ultimo ciclo glaciale (> MIS 4) e, molto probabilmente attribuibile all'ultimo interglaciale (MIS 5e).

La limitata distribuzione del patrimonio genetico del "lineage" del Mare di Ross suggerisce che le aree rifugio di questa popolazione nel Pleistocene superiore dovevano essere molto vicine a questo settore antartico. Inoltre, la relativamente alta frequenza del patrimonio genetico di questa popolazione al margine settentrionale della Terra Vittoria e la sua progressiva diminuzione man mano ci si sposti verso la piattaforma di Ross, al margine meridionale della colonizzazione attuale, a vantaggio della popolazione circumantartica, indica che entrambe le popolazioni avrebbero progressivamente colonizzato le aree costiere del Mare di Ross ma con un comportamento relativamente diversificato, relativamente meno dinamico nel caso della popolazione ancestralmente legata alle aree rifugio al margine esterno del Mare di Ross.

La colonizzazione della Terra Vittoria da parte dei pinguini di Adelia è intimamente legata alla presenza di un'altra importante specie di predatori, gli elefanti marini meridionali, che si sono recentemente rivelati importanti attori nella storia della colonizzazione delle coste antartiche durante l'Olocene (Hall et al., 2006; De Bruyn et al., 2009, 2014).

Una popolazione originaria della lontanissima Isola Macquarie ha colonizzato le coste della Terra Vittoria non appena questo habitat si è reso disponibile, dopo il ritiro dei ghiacciai avvenuto dopo l'UMG (De Bruyn et al., 2009, 2014). Nell'arco di poche generazioni si è sviluppata una popolazione geneticamente molto diversa da quella originale. I fattori che hanno guidato questo evento sono rappresentati dall'assenza stagionale della banchisa, dalla disponibilità di spiagge sabbioso-ghiaiose e ciottolose facilmente raggiungibili e dalla disponibilità di cibo. La variabilità delle condizioni ambientali che rispondono a questi fattori ha peraltro guidato la fluttuazione del numero di siti occupati nel corso dell'Olocene, la variazione delle dimensioni della popolazione e l'abbandono di questo settore antartico nell'ultimo millennio.

Sulla base delle numerose date ${ }^{14} \mathrm{C}$ associate all'occupazione delle colonie di pinguini e di elefanti marini, è evidente che sono esistite nell'Olocene condizioni più o meno favorevoli per i fattori che limitano o favoriscono la presenza di queste due specie animali (Baroni e 
Orombelli, 1994, Hall et al., 2006, Mezgec et al., 2017; Fig. 12). In particolare, la presenza o l'assenza di elefanti marini è condizionata dalla estensione e dalla persistenza del ghiaccio marino e, soprattutto, della banchisa costiera costituita da ghiaccio pluriennale, come evidenziato dall'associazione di diatomee e altri microfossili che si trovano nei sedimenti marini (Mesgec et al., 2017).

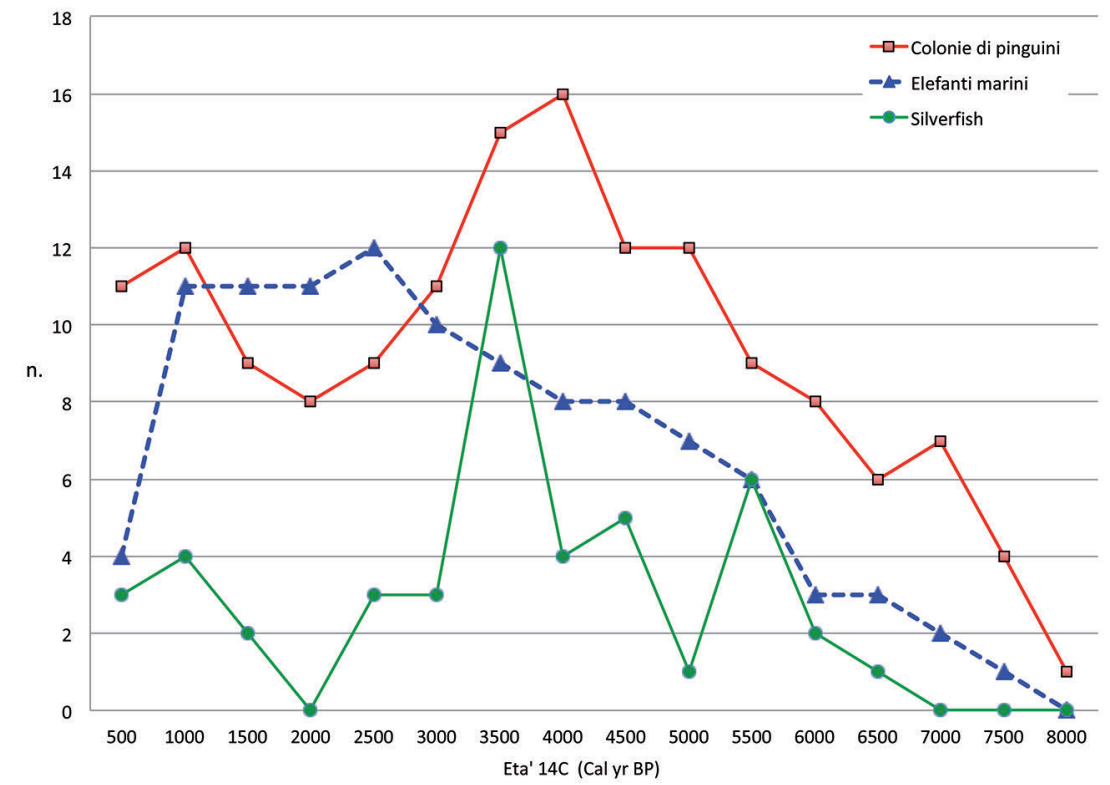

Fig. 12. Distribuzione e frequenza delle colonie di pinguini di Adelia (attuali e relitte), delle colonie di elefanti marini e di resti di Pleurogramma antarcticum (silverfish) da livelli di guano campionati in siti abbandonati di pinguini nella Terra Vittoria ( $i$ dati sono raggruppati per intervalli di 500 anni. (Modificato da Mezgec et al., 2017, con dati inediti di Baroni e Salvatore).

A seguito della deglaciazione completata intorno a 8000 anni fa, una polynia si stabilì al largo di Inexpressible Island nella zona di baia Terra Nova. Tra 8000 e circa 5000 anni elefanti marini e pinguini di Adelia occuparano estesamente le aree deglaciate della Terra Vittoria. Tra 5000 e circa 3000 anni fa si è verificato un periodo particolarmente favorevole allo sviluppo di colonie di pinguini di Adelia, che hanno colonizzato estesi settori costieri, oggi non più occupati ("penguin optimum”, Baroni e Orombelli, 1994; Hall et al., 2004). 
In questo periodo la popolazione di elefanti marini ha subito una forte riduzione, persistendo principalmente nei siti colonizzati più vicini alla polynia di Inexpressible Island.

Tra 2500 e 1100 anni fa, condizioni subantartiche hanno caratterizzato le coste della Terra Vittoria, favorendo una significativa espansione della presenza di elefanti marini, che in quel periodo hanno occupato il maggior numero di colonie. In questo periodo l'estensione della banchisa era ai minimi termini, almeno stagionalmente. Le colonie di pinguini, in questa fase, avrebbero subito una drastica riduzione, sia in termini di colonie occupate sia in termini di dimensioni della popolazione.

La scomparsa delle colonie è avvenuta attraverso un collo di bottiglia che si osserva, su base genetica, intorno a 1000 anni fa (De Bruyn et al., 2014) e che vede l'espulsione degli elefanti marini dal Mare di Ross intorno a 500 anni fa.

In passato, la variabilità delle condizioni ambientali era direttamente associata a condizioni climatiche semplicemente intese in relazione a conditioni di temperatura più o meno elevata. Mezgec et al. (2017) hanno recentemente dimostrato che le condizioni che favoriscono o limitano la presenza di pinguini, elefanti marini e pesci preferenzialmente predati da queste specie (Pleurogramma antarticum) sono in realtà controllati da un più complesso insieme di condizioni ambientali, tra le quali emerge il ruolo delle polynia nel condizionare la produzione di ghiaccio marino e la persistenza di ghiaccio stagionale e pluriennale nelle aree costiere. Complessivamente, i nuovi dati indicano che i venti catabatici sono i principali attori che regolano la formazione del ghiaccio marino (controllando l'efficienza della polynia) e la persistenza della banchisa costiera, mentre i venti geostrofici guidano l'allontanamento e l'estensione del ghiaccio marino nel Mare di Ross, chiarendo che le variazioni ambientali registrate dall'ecosistema marino in Antartide non sono meramente riconducibili a semplici variazioni di temperatura ma vanno considerate in un più ampio contesto.

\section{BIBLIOGRAFIA}

Ainley D.G., 2002. The Adélie penguin: bellwether of climate change, Columbia University Press, New York, 310 pp.

Ainley D.G., Clarke E.D., Arrigo K., Fraser W.R., Kato A., Barton K.J., Wilson P.R., 2005. Decadal-scale changes in the climate and biota of the Pacific sector of the Southern Ocean, 1950's to the 1990s. Antarctic Science, 17, 171-182. 
Ainley D.G., Wilson P.R., Barton K.J., Ballard G., Nur N., Karl B., 1998. Diet and foraging effort of Adélie penguins in relation to pack-ice conditions in the southern Ross Sea. Polar Biology, 20, 311-319.

Anderson J.B., 1999. Antarctic Marine Geology. Cambridge University Press, Cambridge, 289 pp.

Ascenzi A., Segre A., 1971. A new Neanderthal child mandible from an Upper Pleistocene site in Southern Italy. Nature, 233, 280-282.

Barker P.F., Cooper A.K. (Eds.), 1997. Geology, Seismic Stratigraphy of the Antarctic Margin. Part 2. Antarctic Research Series. American Geophysical Union 71, Washington, USA, 187 pp.

Baroni C., 1994. Notes on late-glacial retreat of the Antarctic ice sheet and Holocene environmental changes along the Victoria Land Coast. Memoirs National Institute of Polar Research (Japan). Series C. Earth Sciences, Special Issue, 50, 85-107.

Baroni C., 2013. Climate Change Impacts on Cold Climates. In: Shroder J. (Editor in Chief), Giardino M., Harbo J. (Eds.), Treatise on Geomorphology. Academic Press, San Diego, CA, vol. 8, Glacial and Periglacial Geomorphology, 430-459. doi: 10.1016/B978-0-12-374739-6.00222-0

Baroni C., Hall B.L., 2004. A new Holocene relative sea-level curve for Terra Nova Bay, Victoria Land. Journal of Quaternary Science, 19, 377-396.

Baroni C., Orombelli G., 1991. Holocene raised beaches at Terra Nova Bay, Victoria Land, Antartica. Quaternary Research, 36, 157-177.

Baroni C., Orombelli G., 1994. Abandoned penguin rookeries as Holocene paleoclimatic indicators in Antarctica. Geology, 22, 23-26.

Bart P.J., Anderson J.B., Trincardi F., Shipp S.S., 2000. Seismic data from the Northern basin, Ross Sea, record extreme expansions of the East Antarctic Ice Sheet during the late Neogene. Marine Geology 166, 31-50.

Bentley M.J., Ocofaigh C., Anderson J.B., Conway H., Davies B., Graham A.G.C., Hillenbrand C.D., Hodgson D.A., Jamieson S.R., Larter R.D., Mackintosh A., Smith J.A., Verleyen E., Ackert R.P., Bart P.J., Berg S., Brunstein D., Canals M., Colhoun E.A., Crosta X., Dickens W.A., Domack E., Dowdeswell J.A., Dunbar R., Ehrmann W., Evans J., Favier V., Fink D., Fogwill C.J., Glasser N.F., Gohl K., Golledge N.R., Goodwin I., Gore D.B., Greenwood S.L., Hall B.L., Hall K., Hedding D.W., Hein A.S., Hocking E.P., Jakobsson M., Johnson J.S., Jomelli V., Jones R.S., Klages J.P., Kristoffersen Y., Kuhn G., Leventer A., Licht K., Lilly K., Lindow J., Livingstone S.J., Mass G., McGlone M.S., McKay R.M., Melles M., Miura H., Mulvaney R., Nel W., Nitsche F.O., O’Brien P.E., Post A.L., Roberts S.J., Saunders K.M., Selkirk P.M., Simms A.R., Spiegel C., Stolldorf, T.D., Sugden D.E., van der Putten N., van Ommen T., Verfaillie D., Vyverman W., Wagner B., White D.A., Witus A.E., Zwartz D., 2014. A Community-based Geological Reconstruction of Antarctic Ice Sheet Deglaciation since the Last Glacial Maximum Quaternary Science Reviews, 100, 1-9.

Biuw M., Boehme L., Guinet C., Costa D., Charrassin J.-B., Roquet F., Bailleul F., Meredith M., Thorpe S., Tremblay Y., McDonald B., Park Y.-H., Rintoul S.R., Bindoff N., Goebel M., Crocker D., Lovell P., Nicholson J., Monks F., Fedak M. A., 2007. Variations in behavior, condition of a Southern Ocean top predator in rela- 
tion to in situ oceanographic conditions. Proceedings of the National Academy of Sciences of the United States of America 104 (34), 13705-13710.

Bonfiglio L., Cassoli P.F., Mallegni F., 1986. Neanderthal parietal, vertebrate fauna, and stone artifacts from the Upper Pleistocene deposits of Contrada Ianni di SanCalogero (Catanzaro, Calabria, Italy). American Journal of Physical Anthropology, 70 (2), 241-250.

Borowicz A., McDowall P., Youngflesh C. Sayre-McCord T., Clucas G., Herman R., Forrest S., Rider M., Schwaller M., Hart T., Jenouvrier S., Polito M.J., Singh H., Lynch H.J., 2018. Multi-modal survey of Adélie penguin mega-colonies reveals the Danger Islands as a seabird hotspot. Scientific Reports, 8, 3926. doi.org/10.1038/s41598-018-22313-w

Campbell I.B., Claridge G.C., 1987. Antarctica: Soils, Weathering Processes, Environment. Elsevier, Amsterdam, 368 pp.

Cape Roberts Science Team, 2000. Studies from the Cape Roberts Project, Ross Sea, Antarctica. Initial Report on CRP-3, Terra Antartica 7, 209 pp.

Clark P.U., Mix A.C., 2002. Ice sheets and sea level of the Last Glacial Maximum, Quaternary Science Reviews, 21 (1-3), 1-7. doi: 10.1016/S0277-3791(01)00118-4

Conway H., Hall B.L., Denton G.H., Gades A.M., Waddington E.D., 1999. Past and future grounding-line retreat of the west antarctic ice sheet. Science, 286 (5438), 280-283. doi:10.1126/science.286.5438.280

Croxall J.P., Trathan P.N., Murphy E.J., 2002. Environmental change and Antarctic Seabird populations. Science, 297, 1510-1514.

Dawson, A.G., 1992. Ice Age Earth: Late Quaternary Geology and Climate. Routledge, London, 299 pp.

De Bruyn M., Hall B.L., Chauke L.F., Baroni C., Koch P.L., Hoelzel A.R., 2009. Rapid Response of a Marine Mammal Species to Holocene Climate and Habitat Change. PLoS Genetics, 5 (7), e1000554. doi:10.1371/journal.pgen.1000554

De Bruyn M., Pinsky M.L., Hall B., Koch P., Baroni C., Hoelzel A.R., 2014. Rapid increase in southern elephant seal genetic diversity after a founder event. Proceedings - Royal Society. Biological Sciences, 281, 20133078-20133085. doi: 10.1098/ rspb.2013.3078

Denton G.H., Hughes T.J., 1981. The Last Great Ice Sheets, Wiley, New York, 484 pp.

Denton G.H., Hughes T.J., 2002. Reconstructing the Antarctic ice sheet at the lastglacial maximum. Quaternary Science Reviews 21, 193-202.

Denton G.H., Prentice M.L., Burckle L.H., 1991. Cainozoic history of the Antarcticice sheet. In: Tingey R.J. (Ed.), The geology of Antarctica. Oxford University Press, Oxford, 365-433.

Emslie S.D., 1995. Age and taphonomy of abandoned penguin rookeries in the antarctic peninsula region. Polar Record, 31 (179), 409-418. doi:10.1017/ S0032247400027388

Emslie S.D., 2001. Radiocarbon dates from abandoned penguin colonies in the Antarctic Peninsula region. Antarctic Science 13 (3), 289-295.

Emslie S.D., Coats L., Licht K., 2007. A 45,000 yr record of Adélie penguins and climate change in the Ross Sea, Antarctica. Geology, 35, 61-64.

Emslie S.D., Fraser W., Smith R.C., Walker W., 1998. Abandoned penguin colonies and 
environmental change in the Palmer Station area, Anvers Island, Antarctic Peninsula. Antarctic Science, 10 (3), 257-268. doi:10.1017/s0954102098000352

Emslie S.D., McDaniel J.D., 2002. Adélie penguin diet, climate change during the middle to late Holocene in northern Marguerite Bay, Antarctic Peninsula. Polar Biology 25(3), 222-229.

Emslie S.D., Woehler E.J., 2005. A 9000-year record of Adélie penguin occupation, diet in the Windmill Islands, East Antarctica. Antarctic Science 17 (1), 57-66.

EPICA-Community-Members, 2004. Eight glacial cycles from an Antarctic ice core. Nature 429, 623-628.

EPICA-Community-Members, 2006. One-to-one coupling of glacial climate variability in Greenland and Antarctica. Nature 444, 195-198.

Fairbanks R.A., 1989. 17,000-year glacio-eustatic sea level record: influence of glacial melting rates on the Younger Dryas event and deep-ocean circulation. Nature 342, 637-642. doi: 10.1038/342637a0

Fischer H., Severinghaus J., Brook E., Wolff E., Albert M., Alemany O., Arthern R., Bentley C., Blankenship D., Chappellaz J., Creyts T., Dahl-Jensen D., Dinn M., Frezzotti M., Fujita S., Gallee H., Hindmarsh R., Hudspeth D., Jugie G., Kawamura K., Lipenkov V., Miller H., Mulvaney R., Parrenin F., Pattyn F., Ritz C., Schwander J., Steinhage D., van Ommen T., Wilhelms F., 2013. Where to find 1.5 million yr old ice for the IPICS "Oldest-Ice" ice core. Climate of the Past, 9, 24892505. doi: 10.5194/cp-9-2489-2013

Forcada J., Trathan P.N., 2009. Penguin responses to climate change in the Southern Ocean. Global Change Biology 15, 1618-1630.

Forcada J., Trathan P.N., Reid K., Murphy E.J., Croxall J.P., 2006. Contrasting population changes in sympatric penguin species in association with climate warming. Global Change Biology, 12, 411-423.

Fretwell P., and 59 altri, 2013. Bedmap2: improved ice bed, surface and thickness datasets for Antarctica. Cryosphere 7, 375e393. doi: 10.5194/tc-7-375-2013.

Fuller E., 1999. The Great Auk. Harry N. Abrams, Inc. Publishers, New York, 448 pp.

Gardner N., Hall B., Wehmiller J., 2006. Pre-holocene raised beaches at Cape Ross, southern Victoria Land, Antarctica. Marine Geology, 229 (3-4), 273-284. doi:10.1016/j.margeo.2006.01.006

Grieve S., 1885. The Great Auk, or Garefowl: Its History, Archaeology, Remains. Thomas C. Jack, London, 141 pp.

Hall B.L., Baroni C., Denton G.H., 2004. Holocene relative sea-level history of the Southern VL Coast, Antarctica, Global and Planetary Change, 42, 241-263.

Hall B.L., Hoelzel A.R., Baroni C., Denton G.H., Le Boeuf B.J., Overturf B., Töpf A.L. 2006. Holocene elephant seal distribution implies warmer-than-present climate in the Ross Sea. Proc. National Academy of Sciences of the USA (PNAS), 103, 1021310217.

Harrington H.J., 1960. Adélie penguin rookeries in the Ross Sea region. Notornis 9 (2), $33-39$.

Heine J.C., Speir T.W., 1989. Ornithogenic soils of the Cape Bird Adélie penguin rookeries, Antarctica. Polar Biology 10, 89-99.

Hindell M.A., Sumner M., Bestley S., Wotherspoon S., Harcourt R.G., Lea M., 
Alderman R., McMahon C.R. 2017. Decadal changes in habitat characteristics influence population trajectories of southern elephant seals. Global Change Biology, 23 (12), 5136-5150. doi: 10.1111/gcb.13776

Huybrechts P., 2002. Sea-level changes at the LGM from ice-dynamic reconstructions of the Greenland and Antarctic ice sheets during the glacial cycles. Quaternary Science Reviews 21 (1-3), 203-231.

IPCC, 2007. Climate Change 2007: The Physical Science Basis. Cambridge University Press, Cambridge, 996 pp.

IPCC, 2013. Climate Change 2013: The Physical Science Basis. Cambridge University Press, Cambridge, UK and New York, NY, USA, 1535 pp.

Jones C.W., Risi M.M., Bester M.N., 2020. Local extinction imminent for southern elephant seals Mirounga leonina at their northernmost breeding site, Gough Island-South Atlantic Ocean. Polar Biol 43, 893-897. doi: 10.1007/s00300-02002679-2

Jouzel J., Masson-Delmotte V., Cattani O., Dreyfus G., Falourd S., Hoffmann G., Minster B., Nouet J., Barnola J.M., Chappellaz J., Fischer H., Gallet J.C., Johnsen S., Leuenberger M., Loulergue L., Luethi D., Oerter H., Parrenin F., Raisbeck G., Raynaud D., Schilt A., Schwander J., Selmo E., Souchez, R. Spahni R., Stauffer B., Steffensen J.P., Stenni B., Stocker T.F., Tison J.L., Werner M., Wolff E., 2007. Orbital and millenial Antarctic climate variability over the past 800,000 years. Science 317, 793-796.

Koch P.L., Hall B.L., de Bruyn M., Hoelzel A.R., Baroni C., Salvatore M.C., 2019. Mummified and skeletal southern elephant seals (Mirounga leonina) from the Victoria Land coast, Ross Sea, Antarctica. Marine Mammal Science, 35 (3), 934956. doi: $10.1111 / \mathrm{mms} .12581$

Lambert D.M., Millar C.D., Swaminathan S., Baroni C., 2010. Evolution on a frozen continent. American Scientist, 98, 386-393. doi: 10.1511/2010.86.386

Lambert D.M., Ritchie P.A., Millar C.D., Holland B., Drummond A.J., Baroni C., 2002. Rates of Evolution in Ancient DNA from Adélie Penguins. Science, 295, 2270-2273.

Lambert F., Delmonte B., Petit J.R., Bigler M., Kaufman P.R., Hutterli M., Stocker T.F., Ruth U., Steffensen J.P., Maggi V., 2008. Dust-climate couplings over the past 800,000 years from the EPICA Dome C ice core. Nature 452, 616-619.

Le Boeuf, B.J., Laws R.M., (Eds.), 1994. Elephant Seals: Population Ecology, Behavior, and Physiology. Berkeley, University of California Press, 414 pp.

Lorenzini S., Baneschi I., Fallick A.E., Salvatore M.C., Zanchetta G., Dallai L., Baroni C., 2012. Insights into the Holocene environmental setting of Terra Nova Bay region (Ross Sea, Antarctica) from oxygen isotope geochemistry of Adélie penguin eggshells. The Holocene, 22, 63-69. doi: 10.1177/0959683611409773

Lorenzini S., Olmastroni S., Pezzo F., Salvatore M.C., Baroni C, 2009. Holocene Adélie Penguin diet in Victoria Land, Antarctica. Polar Biology, 32 (7), 1077-1086. doi: 10.1007/s00300-009-0607-4

Lorenzini S., Baroni C., Fallick A.E., Baneschi I., Salvatore M.C., Zanchetta G., Dallai L., 2010. Stable isotopes reveal Holocene changes in the diet of Adélie penguins in Northern Victoria Land (Ross Sea, Antarctica). Oecologia, 164 (4), 911-919. doi: 10.1007/s00442-010-1790-2 
Lynch H.J., LaRue M.A., 2014. First global census of the Adélie penguin. The Auk, 131, 457-466. doi: 10.1642/AUK-14-31.1

Masson-Delmotte V., Stenni B., Pol K., Braconnot P., Cattani O., Falourd S., Kageyama M., Jouzel J., Landais A., Minster B., Barnola J.M., Chappellaz J., Krinner G., Johnsen S., Röthlisberger R., Hansen J., Mikolajewicz U., Otto-Bliesner B., 2010. EPICA Dome C record of glacial and interglacial intensities. Quaternary Science Reviews, 29 (1-2), 113-128. doi: 10.1016/j.quascirev.2009.09.030.

McDaniel J.D., Emslie S.D., 2002. Fluctuations in Ade lie penguin prey size in the mid to late Holocene, northern Marguerite Bay, Antarctic Peninsula. Polar Biology 25 (8), 618-623.

McKay R., Browne G., Carter L., Cowan E., Dunbar G., Krissek L., Naish T., Powell R., Reed J., Talarico F., Wilch T., 2009. The stratigraphic signature of the late Cenozoic Antarctic Ice Sheets in the Ross Embayment. Bulletin of the Geological Society of America 121 (11-12), 1537-1561.

Mezgec K., Stenni B., Crosta X., Masson Delmotte V., Baroni C., Braida M., Ciardini V., Colizza E., Melis R., Salvatore M.C., Severi M., Scarchilli C., Traversi R., Udisti R., Frezzotti M. (2017) - Holocene sea ice variability driven by wind and polynya efficiency in the Ross Sea. Nature Communications, 8, 1-12. doi: 10.1038/s41467017-01455-x

Michel, R., Ferriera, M.C.E., Shaefer, G.R., et al., 2006. Ornithogenic Gelisols (Cryosols) from Maritime Antarctica. Soil Science Society of America Journal 70, 1370-1376

Millar C.D., Dodd A., Anderson J., Gibb G.C., Ritchie P.A., Baroni C., Woodhams M.D., Hendy M.D., Lambert D.M. (2008) - Mutation and Evolutionary Rates in Adélie Penguins from the Antarctic. PLoS Genetics 4(10): e1000209. doi: 10.1371/journal.pgen.1000209

Morlighem M. et al. 2017, IceBridge BedMachine Greenland, Version 3. Boulder, Colorado USA. NASA National Snow and Ice Data Center Distributed Active Archive Center (updated 2018) doi: 10.5067/2CIX82HUV88Y.

Nakada M., Kimura R., Okuno J., Moriwaki K., Miura H., Maemoku H., 2000. Late Pleistocene and Holocene melting history of the Antarctic ice sheet derived from sea-level variations. Marine Geology, 167 (1-2), 85-103.

Naish T., Powell R., Levy R., Wilson G., Scherer R., Talarico F., Krissek L., Niessen F., Pompilio M., Wilson T., Carter L., DeConto R., Huybers P., McKay R., Pollard D., Ross J., Winter D., Barrett P., Browne G., Cody R., Cowan E., Crampton J., Dunbar G., Dunbar, N., Florindo F., Gebhardt C., Graham I., Hannah M., Hansaraj D., Harwood D., Helling D., Henrys S., Hinnov L., Kuhn G., Kyle P., Läufer A., Maffioli P., Magens D., Mandernack K., McIntosh W., Millan C., Morin R., Ohneiser C., Paulsen T., Persico D., Raine I., Reed J., Riesselman C., Sagnotti L., Schmitt D., Sjunneskog C., Strong P., Taviani M., Vogel S., Wilch T., Williams T. 2009. Obliquity-paced pliocene west antarctic ice sheet oscillations. Nature, 458 (7236), 322-328. doi:10.1038/nature07867

Orombelli G., Baroni C., Denton G.H. (1990) - Late Cenozoic glacial history of the Terra Nova Bay Region, northern Victoria Land, Antarctica. Geografia Fisica e Dinamica Quaternaria, 13 (2), 139-163. 
Parks M., Subramanian S., Baroni C., Salvatore M.C., Zhang G., Millar C.D., Lambert D.M., 2015. Ancient population genomics and the study of evolution. Philosophical Transactions Royal Society London Series B: Biological Sciences, 370, 1-10. doi: 10.1098/rstb.2013.0381

Polito M., Emslie S.D., Walker W., 2002. A 1000-year record of Adélie penguin diets in the southern Ross Sea. Antarctic Science, 14, 327-332.

Pollard D., DeConto R.M., 2009. Modelling West Antarctic ice sheet growth, collapse through the past five million years. Nature, 458 (7236), 329-332.

Ritchie P.A., Millar C.D., Gibb G.C., Baroni C., Lambert D.M., 2004. Ancient DNA Enables Timing of the Pleistocene Origin and Holocene Expansion of Two Adélie Penguin Lineages in Antarctica. Molecular Biology and Evolution, 21 (2), 240-248. doi: $10.1093 / \mathrm{molbev} / \mathrm{msh} 012$

Santora J.A., LaRue M.A., Ainley D.G., 2020. Geographic structuring of Antarctic penguin populations. Global Ecology and Biogeography, 1-13. doi: 10.1111/geb.13144

Serjeantson D., 2001. The Great Auk, the Gannett: a Prehistoric Perspective on the Extinction of the Great Auk. International Journal of Osteoarchaeology 11, 43-55.

Shepherd L.D., Millar C.D., Ballard G., Ainley D.G., Wilson P.R., Haynes G.D., Baroni C., Lambert D.M., 2005. Microevolution and mega-icebergs in Antarctica. Proceedings of the National Academy of Sciences of the United States of America (PNAS), 102 (46), 16717-16722. doi:10.1073/pnas.0502281102

Simas F.N.B., Schaefer C.E., Albuquerque Filho, M.R., et al., 2008. Genesis, properties and classification of cryosols from Admiralty Bay, maritime Antarctica. Geoderma 144, 116-122.

Simas, F.N.B., Schaefer, C.E., Melo, V.F., et al., 2007. Ornithogenic cryosols from maritime Antarctica: phosphatization as a soil forming process. Geoderma138, 191-203.

Simmonds M.P., Isaac S.J., 2007. The impacts of climate change on marine mammals: early signs of significant problems. Oryx 41, 19-26. doi: 10.1017/S00306053 07001524

Speir T.W., Cowling J.C., 1984. Orthogenetic soils of the Cape Bird Adelie penguin rookeries, Antarctica. Polar Biology 2, 207-212.

Stokstad, E., 2007. Boom and bust in a Polar hot zone. Science 315 (5818), 1522-1523. doi: 10.1126/science.315.5818.1522.

Subramanian S., Denver D.R., Millar C.D., Heupink T., Aschrafi A., Emslie D.S., Baroni C., Lambert D.M., 2009. High mitogenomic evolutionary rates and time dependency. Trends in Genetics, 25 (11), 482-486. doi: 10.1016/j.tig.2009.09.005

Syroechkovsky E.E., 1959. The role of animals in the formation of primary soils under the conditions of circumpolar regions of the earth (Antarctica). Zoologicheskiy Zhurnal 38, 1770-1775.

Tatur A., Myrcha A., 1989. Soils and vegetation in abandoned penguin rookeries. Proceeding NIPR Symposium Polar Biology 2, 181-189.

Taylor R.H., Wilson P.R., Thomas B.W., 1990. Status and trends of Adélie Penguin populations in the Ross Sea region. Polar Record 26, 293-304.

Ugolini F.C., 1972. Ornithogenic soils of Antarctica, Antarctic Research Series, 20, 181-193.

Wassmann P., Duarte C.M., Agusti S., Sejr M.K., 2011. Footprints of climate change in the Arctic marine ecosystem. Global Change Biology 17 (2), 1235-1249. 
Williams R., McEldowney A., 1990. A guide to the fish otoliths from waters off the Australian Antarctic Territory, ANARE Research Notes 75, Antarctic Division Australia.

Woehler E.J., 1993. The Distribution and Abundance of Antarctic and Subantarctic Penguins. Scientific Committee on Antarctic Research, Cambridge, UK.

Zachos J.C., Pagani M., Sloan L., Thomas E., Billups K., 2001. Trends, rhythms, aberrations in global climate $65 \mathrm{Ma}$ to present. Science 292, 686-693.

Zhu R., Sun L., Yin X., Xie Z., Liu X., 2005. Geochemical evidence for rapid enlargement of a gentoo penguin colony on Barton Peninsula in the maritime Antarctic. Antarctic Science 17, 11-16. 
